\title{
Resource Allocation for Secure Wireless Powered Integrated Multicast and Unicast Services with Full Duplex Self-Energy Recycling
}

\author{
Zheng Chu, Member, IEEE, Fuhui Zhou, Member, IEEE, Pei Xiao, Senior Member, IEEE, Zhengyu \\ Zhu, Member, IEEE, De Mi, Member, IEEE, Naofal Al-Dhahir, Fellow, IEEE, and Rahim Tafazolli, Senior \\ Member, IEEE
}

\begin{abstract}
This paper investigates a secure wireless powered integrated service system with full duplex self-energy recycling. Specifically, an energy-constrained information transmitter (IT), powered by a power station (PS) in a wireless fashion, broadcasts two types of services to all users: a multicast service intended for all users, and a confidential unicast service subscribed to by only one user while protecting it from any other unsubscribed users and an eavesdropper. Our goal is to jointly design the optimal input covariance matrices for the energy beamforming, the multicast service, the confidential unicast service, and the artificial noises from the PS and the IT, such that the secrecy-multicast rate region (SMRR) is maximized subject to the transmit power constraints. Due to the non-convexity of the SMRR maximization (SMRRM) problem, we employ a semidefinite programmingbased two-level approach to solve this problem and find all of its Pareto optimal points. In addition, we extend the SMRRM problem to the imperfect channel state information case where a worst-case SMRRM formulation is investigated. Moreover, we exploit the optimized transmission strategies for the confidential service and energy transfer by analyzing their own rank-one profile. Finally, numerical results are provided to validate our proposed schemes.
\end{abstract}

Index Terms-Wireless powered communication network, physical layer security, full duplex, self-energy recycling, secrecymulticast performance tradeoff.

\section{INTRODUCTION}

Achieving high data rates and reliable transmission are key objectives for the forthcoming fifth generation $(5 \mathrm{G})$ wireless communication networks, which are motivated by a growing consumers' desire for high-quality multimedia wireless devices (WDs) (such as 4k hand-held devices and 3D augmented reality) [1]. In addition, high-quality transmission services include: a multicast service, which is subscribed to by all WDs, and a confidential unicast service, which is subscribed to by a dedicated WD to prevent unauthorized access from the unsubscribed WDs and the dedicated eavesdroppers. A heuristic approach is to combine these two services into one

Z. Chu, P. Xiao, D. Mi, and R. Tafazolli are with Institute of Communication Systems, University of Surrey, Guildford GU2 7XH, United Kingdom. (Email: zheng.chu@surrey.ac.uk; p.xiao@surrey.ac.uk; d.mi@surrey.ac.uk; r.tafazolli@surrey.ac.uk)

F. Zhou is with the School of Information Engineering, Nanchang University, Nanchang 330031, China. (Email: zhoufuhui@ieee.org)

Z. Zhu is with the School of Information Engineering, Zhengzhou University, Zhengzhou 450001, China. (Email: zhuzhengyu6@gmail.com)

N. Al-Dhahir is with the Department of Electrical and Computer Engineering, University of Texas at Dallas, Dallas, TX 75080 USA (e-mail: aldhahir@utdallas.edu). integral service over one transmission time block, which is defined as physical layer service integration (PHY-SI) [2], [3]. In a PHY-SI system, these two coexisting services will share the same radio resources by exploiting the physical characteristics of wireless channels to significantly enhance the spectral efficiency. However, in general, the confidential and multicast (or public) services must be available to different user groups to satisfy their own demands [3]. Thus, it is critical to guarantee reliable transmission for the confidential unicast service without sacrificing the quality of the multicast service.

As a result, physical layer multicasting and physical layer security have received increasing attention in the literature in recent years. In a multi-antenna multicasting system, the transmit beamforming/precoding is typically designed to ensure efficient transmission of the common messages that all receivers can decode to maximize the sum-rate while maintaining the desired quality-of-service (QoS) level for all users [4]-[6]. Due to the vulnerable nature of the wireless broadcast channel to eavesdropping, physical layer security techniques are becoming increasingly important. They achieve high secrecy performance without secret key distribution and management that may lead to security vulnerability in wireless channels. The key feature of physical layer security is that the channel for the legitimate user must be better than the eavesdropper's channel to guarantee a positive secrecy rate that is defined as the mutual information difference between the legitimate user's channel and the eavesdropper's channel to the transmitter [7]. Recently, various secure transmission strategies against eavesdropping have been developed based on information-theoretical studies [8], [9], where multi-antenna wiretap channels have been investigated to take advantage of the additional degrees of freedom (DoF) and diversity gains. The existing techniques in multi-antenna secrecy channels aim to design the optimal transmit beamforming vectors, and to introduce more interference to degrade the eavesdroppers' link (i.e., artificial noise (AN) and cooperative jammer (CJ)), thus improving the achievable secrecy rate in multi-antenna secrecy channels [10]-[13].

Due to the explosive growth of the high-data-rate multimedia wireless services in $5 \mathrm{G}$ networks, more energies are consumed in battery-powered devices to meet the demands of various transmission services. How to prolong the lifetime of the energy-limited devices is another crucial problem, and one promising solution is microwave wireless energy transfer 
(WET) [14]. In wireless-powered communication networks (WPCNs), wireless transceivers are wirelessly charged by the power transmitter [15], [16]. Unlike the traditional batterypowered networks, WPCN can effectively reduce the operational cost to realize renewable charging without battery replacement or recharging. In addition, the energy released by the radio frequency signal in the WET network is adjustable for providing a stable energy supply to satisfy different physical constraints and service requirements [16]. In WPCNs, a well-known protocol, named "harvest-then-transmit" was proposed in [17], where the wireless users are wirelessly charged by a hybrid access-point (AP) in the downlink (DL), and then they use the harvested energy to send the information signal to the AP in the uplink (UL). Recently, a dedicated WET network was proposed to deploy multiple power beacons (PBs) near a wireless information transfer (WIT) network [18], [19]. It is feasible to deploy the PBs densely to guarantee network coverage due to the low cost of the PBs deployment [20]. In [21], the power consumption model is evaluated to quantify the energy efficiency (EE) of a wireless network, which proposed an energy efficiency evaluation framework $\left(\mathrm{E}^{3} \mathrm{~F}\right)$ for a variety of base station (BS) models. It is applied to quantify the EE performance of the downlink in a $3 \mathrm{GPP}$ LTE radio access network. Integrating physical layer security with WPCN can improve security and sustainable energy efficiency in a multicasting system [1]. Multiple PBs are deployed to provide wireless energy to support the secure multicasting transmission, which solves the constrained energy problem and guarantees secure transmissions simultaneously. However, the above works mainly focus on the half-duplex (HD) systems with wireless information and power transfer in orthogonal time slots, which typically leads to low spectrum utilization.

As a result, full-duplex (FD) wireless systems have gained increasing interest due to their potential to double the spectral efficiency by simultaneous signal transmission and reception over the same frequency band [22]-[26]. In [22], a full-duplex wireless communication system was investigated, where same band simultaneous bidirectional communication is achieved via cancellation of the self-interfering signal. By exploiting off-the-shelf multiple-input multiple-output (MIMO) radios, the experimental results characterize the suppression performance of three self-interference cancellation mechanisms, which combine different mixes of analog and digital cancellation. In [23], a FD multiuser MIMO system was investigated, where a BS bidirectionally communicates with multiple users in the downlink (DL) and uplink (UL) channels on the same system resources. The spectral efficiency (SE) and EE are maximized by jointly designing the precoders in DL and UL. A novel energy recycling single-antenna FD radio is developed, which includes a power divider and an energy harvester added between the circulator and the receiver chain [24]. The proposed self-energy recycling architecture can bring benefits in terms of spectral efficiency and energy consumption. In [25], [26], a novel multicarrier non-orthogonal multiple access (MC-NOMA) system is investigated by considering a FD BS. The optimal joint power and subcarrier allocation algorithm is developed to maximize the weighted sum throughput [25], while, the robustness and security performance is studied in
FD MISO MC-NOMA systems by taking into account the imperfectness of the eavesdroppers' channel state information (CSI). Furthermore, the FD technique was introduced in relay systems [27]-[29], where the energy-constrained relay harvests energy with self-energy recycling, and forwards the information signal to the destination node simultaneously. Secure FD WPCN systems were investigated in [30], [31], where the secrecy FD information and power transmission was investigated with self-energy recycling. Particularly, in [30], the optimal solution for the transmit beamforming vector was derived in closed-form. A robust secure beamforming scheme was developed based on the imperfect CSI case [31]. In [32], the power control problems for in-band FD $\mathrm{EH}$ wireless networks were formulated to minimize the sum transmit power subject to the QoS constraint and maximize the sum throughput. These two problems were solved via the distributed power control schemes for controlling the uplink transmit power levels by the users and the downlink energy harvesting signal power by the hybrid $\mathrm{BS}$.

Currently, various existing works focus on the PHY-SI system. Specifically, the fundamental limit on the achievable rate region in a PHY-SI system was investigated subject to the secrecy constraint in [33]. The optimal integration of both multicast and confidential services was derived in a discrete memoryless broadcast channel. In [34]-[36], the results were extended to the MIMO Gaussian broadcast case. Moreover, the PHY-SI strategy has been applied to bidirectional relay networks in [37], where the broadcast, multicast and confidential services are integrated at a relay node to establish bidirectional communications. The work in [38] deduced the achievable secrecy rate region under channel uncertainty in a compound broadcast channel, which represents a robust PHY-SI transmit strategy. However, the aforementioned works only tackle the PHY-SI problem from information theoretic aspects, where the main goal is to derive capacity results or to analyze coding schemes that achieve certain rate regions [2]. To pave the road for practical implementation, it is also important to investigate PHY-SI from signal processing aspects, and identify the optimal transmit strategy for the transmitted integrated services to maximize the achievable secrecy rate regions [2], [3]. More importantly, it is necessary to improve the spectral and energy utilization of the IT. Thus, integrating the FD self-energy recycling with PHY-SI is a practical and promising idea, where by harvesting energy from the energy source and self-energy recycling, the FD IT serves the integrated confidential unicast and multicast services to the users. To the best of our knowledge, there is no existing work that has investigated this integration. The above-mentioned research gap motivates our development of the techniques presented in this paper.

In this paper, we investigate a secure wireless powered service integration system with FD self-energy recycling. We formulate a secrecy-multicast rate region maximization (SMRRM) problem subject to the transmit power and the energy harvesting constraints by incorporating perfect or imperfect CSI. Our goal is to jointly design the optimal input covariance matrices for the energy beamforming, the multicast service, the confidential service, and the ANs from the PS and FD IT. The 
contributions of this paper are highlighted as follows:

1) Secure wireless powered integrated service with FD selfenergy recycling: We consider a wireless powered integrated service system with FD self-energy recycling. Specifically, a FD IT, powered by a PS, provides the integrated multicast and confidential unicast service to the users in the presence of a multi-antenna external eavesdropper. The AN matrices are injected at the PS and the FD IT to guarantee the confidential unicast information transmission. The proposed system improves the secrecy-multicast performance tradeoff by utilizing wireless charge and self-energy recycling.

2) Optimized designs based on perfect or imperfect CSI: Second, we develop a joint optimization scheme of ANembedded confidential unicast and multicast services as well as an energy beamformer to maximize the SMRR subject to transmit power and energy harvesting constraints. Due to non-convexity of this optimization problem, we first transform the SMRRM problem to an equivalent scalar optimization problem for the perfect CSI case. Subsequently, we propose a semidefinite programming (SDP) two-level optimization problem to further recast it into a convex form. Specifically, the inner level can be reformulated as an SDP, whereas the outer level is a single-variable optimization problem, which can be handled via a one-dimensional search. In addition, we extend the SMRRM problem to the imperfect CSI case, where the worst-case formulation is investigated. By applying the quadratic matrix inequality (QMI) technique, the worst-case SMRRM counterpart can also be recast as the SDP.

3) Implementation efficiency analysis: Moreover, we investigate the feasibility of secrecy-multicast performance tradeoff and present the optimal solution analysis for the proposed scheme. First, we exploit the Pareto optimal points for our formulated SMRRM problem. We also characterize the rank-profile of the optimal solution to the SMRRM problem, and derive the condition for the optimal strategy of the confidential service. We show that the reformulated problem yields a rank-one solution for the optimal confidential covariance matrix. In addition, we analyze the optimal solution of the reformulated problem with the optimal confidential strategy by constructing a new solution. Furthermore, we show that the energy beamforming matrix is rank-one such that the optimal energy transfer strategy can be achieved.

The rest of this paper is organized as follows: Section II describes our system model. The optimization designs for the perfect CSI scenario are presented in III. Section IV extends our results to the imperfect CSI scenario. Numerical results are provided in Section $\mathrm{V}$ and the paper is concluded in Section VI. The summary of notations will be presented in Table I.

\section{SYSTEM MODEL}

In this section, we consider a secure wireless powered integrated service system as shown in Fig. 1, which consists of one power station (PS), one information transmitter (IT), $K$ users, and one external eavesdropper. The PS is equipped

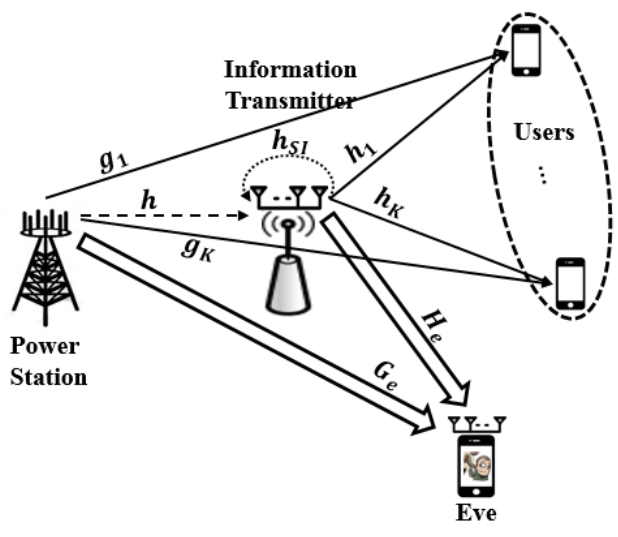

Fig. 1: A secure wireless powered FD integrated service system.

with $N_{P S}$ transmit antennas, and the IT consists of $N_{I T}+1$ antennas with $N_{I T}$ for integrated information service transmission, and one for energy harvesting. All users are equipped with a single antenna, and the eavesdropper is equipped with $N_{E}$ antennas. The IT, powered by the stable wireless charge from the PS, broadcasts the integrated services (i.e., multicast and confidential unicast services) to all users. Meanwhile, the IT adopts the FD scheme to collect energy from the PS and self-interference, respectively. It is assumed that the multicast service has been ordered by all users, and only user 1 further subscribed to the confidential service. ${ }^{1}$ To enhance the security of the confidential service, both the PS and FD IT inject ANs to interfere with the unauthorized users (i.e., potential eavesdroppers, user 2 to user $K$ ) and the dedicated eavesdropper. A summary of notations for our system model is provided in Table II. The received signals at the IT, user $k$, and the eavesdropper are given by

$$
\begin{aligned}
& y_{\mathrm{IT}}=\mathbf{h}^{H} \mathbf{v}+\mathbf{h}^{H} \mathbf{z}_{P S}+\mathbf{h}_{S I}^{H} \mathbf{x}+n_{I T}, \\
& y_{k}=\mathbf{h}_{k}^{H} \mathbf{x}+\mathbf{g}_{k}^{H} \mathbf{z}_{P S}+n_{k}, \mathbf{y}_{e}=\mathbf{H}_{e}^{H} \mathbf{x}+\mathbf{G}_{e}^{H} \mathbf{z}_{P S}+\mathbf{n}_{e},
\end{aligned}
$$

where $\mathbf{v} \in \mathbb{C}^{N_{P S} \times 1}$ and $\mathbf{z}_{P S} \in \mathbb{C}^{N_{P S} \times 1}$ are the energy signal and the AN signal sent by the PS, respectively, which are assumed to be pseudo-random with covariance matrices $\mathbf{V} \in \mathbb{H}_{+}^{N_{P S}}$ and $\mathbf{Z}_{P S} \in \mathbb{H}_{+}^{N_{P S}}$, i.e., $\mathbf{v} \sim \mathcal{C N}(\mathbf{0}, \mathbf{V})$ and $\mathbf{z}_{P S} \sim \mathcal{C N}\left(\mathbf{0}, \mathbf{Z}_{P S}\right)$. In addition, $\mathbf{x} \in \mathbb{C}^{N_{I T} \times 1}$ is the transmitted signal vector from the FD IT which consists of three independent components, i.e., $\mathbf{x}=\mathbf{w}_{0}+\mathbf{w}_{p}+\mathbf{z}_{I T}$, where $\mathbf{w}_{0} \in \mathbb{C}^{N_{I T} \times 1}$ is the multicast message intended for all users, $\mathbf{w}_{p} \in \mathbb{C}^{N_{I T} \times 1}$ is the confidential message subscribed to by user 1 , and $\mathbf{z}_{I T} \in \mathbb{C}^{N_{I T} \times 1}$ is the $\mathrm{AN}$ generated by the FD IT, where we assume $\mathbf{w}_{0} \sim \mathcal{C N}\left(\mathbf{0}, \mathbf{W}_{0}\right)$, $\mathbf{w}_{p} \sim \mathcal{C N}\left(\mathbf{0}, \mathbf{W}_{p}\right)$, and $\mathbf{z}_{I T} \sim \mathcal{C N}\left(\mathbf{0}, \mathbf{Z}_{I T}\right)$. Moreover, $n_{I T} \sim \mathcal{C N}\left(0, \sigma_{I T}^{2}\right), n_{k} \sim \mathcal{C N}\left(0, \sigma_{k}^{2}\right)$, and $\mathbf{n}_{e} \sim \mathcal{C N}\left(0, \sigma_{e}^{2} \mathbf{I}\right)$ are the additive white Gaussian noise (AWGN) signals at the receive antenna of the FD IT, user $k$, and the eavesdropper, respectively. Based on (1a), the amount of harvested energy by

\footnotetext{
${ }^{1}$ In this paper, it is assumed that only one user subscribes to the confidential service during a single time block. In practice, this corresponds to the scenario where the confidential service can be broadcast to all users in a round-robin manner to enhance the reliability of the confidential service and to reduce the operational complexity at the FD IT [3].
} 
TABLE I: Notations

\begin{tabular}{cc}
\hline Notations & Descriptions \\
\hline$(\cdot)^{H}$ & Conjugate transpose \\
$\|*\|$ & Euclidean norm of a vector \\
$\operatorname{Tr}(\cdot)$ & Trace of a matrix \\
$\mathbf{I}$ & Identity matrix with appropriate size \\
$\mathbb{E}\{\cdot\}$ & Statistical expectation of a random variable \\
$(\cdot)^{-1}$ & Inverse of a matrix \\
$\mathbf{A} \succeq \mathbf{0}$ & $\mathbf{A}$ is a positive semidefinite matrix \\
{$[x]^{+}$} & $\max \{x, 0\}$ \\
$|\mathbf{A}|$ & Determinant of $\mathbf{A}$ \\
$\mathbf{x} \sim \mathcal{C} \mathcal{N}(\mu, \Omega)$ & $\mathbf{x}$ is a complex circular Gaussian random variable with mean $\mu$ and covariance $\boldsymbol{\Omega}$ \\
\hline
\end{tabular}

TABLE II: Notations of System Model

\begin{tabular}{cccc}
\hline Notations & Descriptions & Notations & Descriptions \\
\hline$K$ & Number of users & $\mathbf{h} \in \mathbb{C}^{N_{P S} \times 1}$ & Channel vector from PS to FD IT \\
$N_{P S}$ & Number of transmit antennas at PS & $\mathbf{g}_{k} \in \mathbb{C}^{N_{P S} \times 1}$ & Channel vector from PS to user $k$ \\
$N_{I T}$ & Number of transmit antennas at FD IT & $\mathbf{G}_{e} \in \mathbb{C}^{N_{P S} \times N_{E}}$ & Channel matrix from PS to eavesdropper \\
$N_{E}$ & Number of receive antennas at eavesdropper & $\mathbf{h}_{k} \in \mathbb{C}^{N_{I T} \times 1}$ & Channel vector from FD IT to user $k$ \\
$\mathcal{K}=\{1, \ldots, K\}$ & Index set of all users & $\mathbf{H}_{e} \in \mathbb{C}^{N_{I T} \times N_{E}}$ & Channel matrix from FD IT to eavesdropper \\
$\overline{\mathcal{K}}=\mathcal{K} /\{1\}$ & Index set of unauthorized users & $\mathbf{h}_{S I} \in \mathbb{C}^{N_{I T} \times 1}$ & Self-loop channel vector. \\
\hline
\end{tabular}

FD IT ${ }^{2}$ is given by $E_{I T}=\xi \mathbb{E}\left(\left|\mathbf{h}^{H} \mathbf{v}+\mathbf{h}^{H} \mathbf{z}_{P S}+\mathbf{h}_{S I}^{H} \mathbf{x}\right|^{2}\right)=$ $\xi\left[\mathbf{h}^{H}\left(\mathbf{V}+\mathbf{Z}_{P S}\right) \mathbf{h}+\mathbf{h}_{S I}^{H}\left(\mathbf{W}_{0}+\mathbf{W}_{p}+\mathbf{Z}_{I T}\right) \mathbf{h}_{S I}\right]$, where $0<$ $\xi \leq 1$ denotes the energy conversion efficiency at the FD IT. $^{3}$ Thus, the transmit power at the FD IT should satisfy $\operatorname{Tr}\left(\mathbf{W}_{0}+\mathbf{W}_{p}+\mathbf{Z}_{I T}\right) \leq P_{I T}+E_{I T}$, where $P_{I T} \geq 0$ denotes the constant energy, which may represent the residual energy at the FD IT from the information transmission at the previous time block or energy obtained from the other power sources. On the other hand, the achievable rates (i.e., multicast rate and secrecy rate) at the FD IT are exploited according to (1b). The multicast rate at user $k$ is given by

$R_{c o m, k}=\log _{2}\left(1+\frac{\mathbf{h}_{k}^{H} \mathbf{W}_{0} \mathbf{h}_{k}}{\mathbf{h}_{k}^{H}\left(\mathbf{W}_{p}+\mathbf{Z}_{I T}\right) \mathbf{h}_{k}+\mathbf{g}_{k}^{H} \mathbf{Z}_{P S} \mathbf{g}_{k}+\sigma_{k}^{2}}\right)$,

and the secrecy rate of user 1 can be written as

$$
R_{\text {sec }}=\left[R_{1}-\max \left\{\max _{k \in \mathcal{K}} R_{k}, R_{e}\right\}\right]^{+},
$$

where

$$
\begin{aligned}
& R_{1}=\log _{2}\left(1+\frac{\mathbf{h}_{1}^{H} \mathbf{W}_{p} \mathbf{h}_{1}}{\mathbf{h}_{1}^{H} \mathbf{Z}_{I T} \mathbf{h}_{1}+\mathbf{g}_{1}^{H} \mathbf{Z}_{P S} \mathbf{g}_{1}+\sigma_{1}^{2}}\right), \\
& R_{k}=\log _{2}\left(1+\frac{\mathbf{h}_{k}^{H} \mathbf{W}_{p} \mathbf{h}_{k}}{\mathbf{h}_{k}^{H} \mathbf{Z}_{I T} \mathbf{h}_{k}+\mathbf{g}_{k}^{H} \mathbf{Z}_{P S} \mathbf{g}_{k}+\sigma_{k}^{2}}\right), k \in \overline{\mathcal{K}}, \\
& R_{e}=\log _{2}\left|\mathbf{I}+\left(\sigma_{e}^{2} \mathbf{I}+\mathbf{H}_{e}^{H} \mathbf{Z}_{I T} \mathbf{H}_{e}+\mathbf{G}_{e}^{H} \mathbf{Z}_{P S} \mathbf{G}_{e}\right)^{-1} \mathbf{H}_{e}^{H} \mathbf{W}_{p} \mathbf{H}_{e}\right| .
\end{aligned}
$$

Note that the second term of (3) denotes the maximum eavesdropping rate from the potential eavesdroppers (i.e., user $k, k \in \overline{\mathcal{K}})$ and the external eavesdropper. It guarantees reliable transmission for the secrecy unicast message intended to user 1. We define $\bar{R}_{\text {com }}$ and $\bar{R}_{\text {sec }}$ as the achievable rate regions with the multicast and confidential services, respectively. Thus,

\footnotetext{
${ }^{2}$ Different from the works in [25], [26], where the FD BS typically employed sophisticated SIC techniques, in our paper, the signal from the transmit antenna of the FD IT enables the self-energy recycling. Specifically, the loop energy that is employed for integrated information transmission by the FD IT can be collected and reused in addition to the dedicated energy powered by the PS.

${ }^{3}$ Note that the energy harvested from the noise at the FD IT is ignored since it is usually small.
}

an achievable secrecy-multicast rate region is given as the set of nonnegative rate region pairs $\left(\bar{R}_{c o m}, \bar{R}_{s e c}\right)$ satisfying the following constraints [7], [34]:

$$
\begin{aligned}
\bar{R}_{\text {com }} & \leq \min _{k \in \mathcal{K}} R_{\text {com }, k}, \\
\bar{R}_{\text {sec }} & \leq R_{1}-\max \left\{\max _{k \in \overline{\mathcal{K}}} R_{k}, R_{e}\right\} .
\end{aligned}
$$

Remark 1: Note that the secrecy-multicast rate region (4) reveals that all users first need to decode the common multicast message by treating the confidential message as interference (c.f. (2)). Then, the first user further subscribes to the secrecy transmission link for its exclusive confidential service, in which there is no interference from the multicast message. In practice, the multicast service is typically known as regular service, whereas the confidential service is known as the premier service. The regular service must guarantee the signal quality of the premier service, but not vice versa.

Remark 2: In this paper, we assume that a wireless-powered FD IT performs the integrated confidential unicast and multicast service, which is termed as information/energy fullduplexing [31]. Specifically, this FD IT can be powered by the PS due to a limited-power supply. Unlike conventional information/information full-duplexing, which typically requires sophisticated self-interference cancellation (SIC) techniques, the signal leakage from the transmit antennas of the wireless powered FD IT is in fact beneficial, since it enables selfenergy recycling, i.e., part of the energy (loop energy) that is employed for information transmission by the FD IT can be harvested and reused in addition to the dedicated energy transmitted by the PS [30], [31], [39].

\section{The SMRRM PRoblem BASEd on PeRfect CSI}

In this section, we formulate the achievable SMRRM problem subject to the transmit power constraints at the PS and the FD IT, which is based on the assumption that all CSIs are 
available at the PS and the FD IT. ${ }^{4}$ This problem is a vector optimization problem, with a cone $K=K^{*}=\mathbb{R}_{+}^{2}$, which is given by

$$
\begin{aligned}
& \max _{\substack{\mathbf{V}, \mathbf{Z}_{P S}, \mathbf{W}_{0}, \mathbf{W}_{p} \\
\mathbf{Z}_{I T}, \bar{R}_{c o m}, \bar{R}_{s e c}}} \bigcup_{\mathbb{R}_{+}^{2}}\left(\bar{R}_{\text {com }}, \bar{R}_{\text {sec }}\right), \quad \text { s.t. }(4), \\
& \operatorname{Tr}\left(\mathbf{V}+\mathbf{Z}_{P S}\right) \leq P_{P S}, \\
& \operatorname{Tr}\left(\mathbf{W}_{0}+\mathbf{W}_{p}+\mathbf{Z}_{I T}\right) \leq P_{I T} \\
& \quad+\xi\left[\mathbf{h}^{H}\left(\mathbf{V}+\mathbf{Z}_{P S}\right) \mathbf{h}+\mathbf{h}_{S I}^{H}\left(\mathbf{W}_{0}+\mathbf{W}_{p}+\mathbf{Z}_{I T}\right) \mathbf{h}_{S I}\right], \\
& \left([\mathbf{V}]_{n, n}+\left[\mathbf{Z}_{P S}\right]_{n, n}\right) \leq p_{P S}^{n}, \forall n \in\left\{1, \ldots, N_{P S}\right\} \\
& \left(\left[\mathbf{W}_{0}\right]_{m, m}+\left[\mathbf{W}_{p}\right]_{m, m}+\left[\mathbf{Z}_{I T}\right]_{m, m}\right) \leq p_{I T}^{m}, \\
& \quad \forall m \in\left\{1, \ldots, N_{I T}\right\} \\
& \quad \mathbf{V}, \mathbf{Z}_{P S}, \mathbf{W}_{0}, \mathbf{W}_{p}, \mathbf{Z}_{I T} \succeq \mathbf{0},
\end{aligned}
$$

where $P_{P S}$ is the maximum available transmit power at the PS, while $p_{P S}^{n}$ and $p_{I T}^{m}$ denote the transmit power limits of the $n$-th and $m$-th antenna at the PS and the FD IT, respectively. Note that the constraints $(5 \mathrm{c})$ and $(5 \mathrm{~d})$ denote the per-antenna power constraints. Problem (5) is a bi-objective optimization problem, where the two objectives are $\bar{R}_{\text {com }}$ and $\bar{R}_{\text {sec }}$. The basic idea to solve this problem is to maximize their weighted sum by searching for the weight factor. However, this method may not find all of the Pareto optimal points [41]. ${ }^{5}$ In this paper, we develop a novel approach to find all Pareto optimal points of (5). Specifically, we first fix the variable $\bar{R}_{c o m}$ as a constant such that (5) is reduced to the maximization of $\bar{R}_{s e c}$. Thus, problem (5) can be simplified into the following secrecy rate maximization (SRM) problem

$$
f^{\text {opt }}\left(\bar{R}_{\text {com }}\right)=\max _{\substack{\mathbf{V}, \mathbf{Z}_{P S} \\ \mathbf{W}_{0}, \mathbf{W}_{p}, \mathbf{Z}_{I T}}} R_{1}-\max \left\{\max _{k \in \overline{\mathcal{K}}} R_{k}, R_{e}\right\},
$$

s.t. (4a), (5a), (5b), (5e),

$\operatorname{Tr}\left[\boldsymbol{\Xi}_{n}\left(\mathbf{V}+\mathbf{Z}_{P S}\right)\right] \leq p_{P S}^{n}, n \in\left\{1, \ldots, N_{P S}\right\}$,

$$
\operatorname{Tr}\left[\boldsymbol{\Lambda}_{m}\left(\mathbf{W}_{0}+\mathbf{W}_{p}+\mathbf{Z}_{I T}\right)\right] \leq p_{I T}^{m}, m \in\left\{1, \ldots, N_{I T}\right\},
$$

where $\boldsymbol{\Xi}_{n}=\mathbf{r}_{n} \mathbf{r}_{n}^{H}$ and $\boldsymbol{\Lambda}_{m}=\mathbf{r}_{m} \mathbf{r}_{m}^{H}$ are the given antenna design parameters to adjust each antenna power budget. For instance, $\mathbf{r}_{n}$ is the $n$-th unit vector, i.e., $\left[\mathbf{r}_{n}\right]_{i}=1$, for $n=i$, and $\left[\mathbf{r}_{n}\right]_{j}=0$, for $n \neq i$. The specific applications of perantenna power constraints have been already described in [11], [12]. From (6), it follows that $\bar{R}_{\text {com }}$ is fixed as a pre-defined requirement of the multicast rate. When $\bar{R}_{c o m}=0$, problem

\footnotetext{
${ }^{4}$ In this paper, it is first assumed that perfect CSI is available on the links from the PS and the FD IT. This is due to the fact that each user in this WPCN PHY-SI network has the registration for subscribing to the multicast service. During this registration or lease renewal, these users are required to feedback their own CSI without noise, which could be achieved by using a low-rate transmission with suitable quantization schemes [3]. In addition, the CSIs of the eavesdropper are assumed to be perfectly known at the PS and the FD IT, which can be achieved through different methods such as the local oscillator power leakage from the eavesdropper receiver's RF frontend [40]. Nonetheless, considering the impact of channel uncertainty, we will also investigate the case of imperfect CSI at the PS and the FD IT in Section IV.

${ }^{5}$ Note that different multi-objective problems were developed in [42], [43], where a weighted Tchebycheff method was employed for investigating the tradeoff between multi-objective functions. However, this method solves multiple optimization problems which is time consuming. In addition, this method may not be applicable to our work, since it would yield a nonconvex scalar optimization problem if used to solve our problem such that it may not find the complete Pareto optimal set [3].
}

(6) becomes a conventional AN-aided SRM problem for a secure WPCN system with FD self-recycling, similar to [31]. Note that the secrecy transmission will be terminated provided that $\bar{R}_{\text {com }}$ is set to an upper bound $\bar{R}_{\text {com }}^{\max }$, which is achieved by solving the following problem

$$
\begin{aligned}
\bar{R}_{\text {com }}^{\max }= & \arg \max _{\mathbf{W}_{0} \succeq \mathbf{0}} \min _{k \in \mathcal{K}} \log _{2}\left(1+\frac{\mathbf{h}_{k}^{H} \mathbf{W}_{0} \mathbf{h}_{k}}{\sigma_{k}^{2}}\right), \\
\text { s.t. } & \operatorname{Tr}\left[\left(\mathbf{I}-\xi \mathbf{h}_{S I} \mathbf{h}_{S I}^{H}\right) \mathbf{W}_{0}\right] \leq P_{I T}+\xi P_{P S}\|\mathbf{h}\|^{2}, \\
& \operatorname{Tr}\left[\mathbf{\Lambda}_{m} \mathbf{W}_{0}\right] \leq p_{I T}^{m}, m \in\left\{1, \ldots, N_{I T}\right\} .
\end{aligned}
$$

It can be easily verified that the constraints in (7b) are obtained from the constraints (5b) and (6b), respectively. From (7), $\bar{R}_{c o m}^{\max }$ can be interpreted as the multicast capacity, which is an upper bound on $\bar{R}_{\text {com }}$. It is easily verified that (7) can be solved by using an SDP reformulation [41]. Now, a question arises whether problem (6) can guarantee a complete inclusion of the Pareto optimal solutions of (5).

Theorem 1: The achievable rate pair $\left(\bar{R}_{c o m}, f\left(\bar{R}_{c o m}\right)\right)$ is a Pareto optimal point of (5), and all Pareto optimal points of (5) can be achieved by solving problem (6) with different $\bar{R}_{\text {com }}$ lying within the interval $\left[0, \bar{R}_{\text {com }}^{\max }\right]$.

Proof: This proof is similar to the proof of [3, Theorem 1]. We omit the details of this proof due to space limitation.

By applying Theorem 1, we can claim that the Pareto optimal solution of (5) can be achieved by solving problem (6) with $\bar{R}_{\text {com }} \in\left[0, \bar{R}_{\text {com }}^{\max }\right]$. Next, we will exploit the optimal solution of problem (6).

\section{A. Solution of The Optimization Problem (6)}

Problem (6) is still nonconvex. In order to address this issue, we rewrite (6) as

$$
\begin{gathered}
f^{\text {opt }}\left(\bar{R}_{\text {com }}\right)=\max _{\substack{\mathbf{V}, \mathbf{Z}_{P S}, \mathbf{W}_{0} \\
\mathbf{W}_{p}, \mathbf{Z}_{I T}, \alpha \geq 1}} R_{1}-\log _{2}(\alpha) \\
\text { s.t. } \max _{k \in \overline{\mathcal{K}}} \log _{2}\left(1+\frac{\mathbf{h}_{k}^{H} \mathbf{W}_{p} \mathbf{h}_{k}}{\mathbf{h}_{k}^{H} \mathbf{Z}_{I T} \mathbf{h}_{k}+\mathbf{g}_{k}^{H} \mathbf{Z}_{P S} \mathbf{g}_{k}+\sigma_{k}^{2}}\right) \leq \log _{2}(\alpha), \\
\log _{2}\left|\mathbf{I}+\left(\sigma_{e}^{2} \mathbf{I}+\mathbf{H}_{e}^{H} \mathbf{Z}_{I T} \mathbf{H}_{e}+\mathbf{G}_{e}^{H} \mathbf{Z}_{P S} \mathbf{G}_{e}\right)^{-1} \mathbf{H}_{e}^{H} \mathbf{W}_{p} \mathbf{H}_{e}\right| \\
\leq \log _{2}(\alpha), \\
\mathbf{h}_{k}^{H}\left[\mathbf{W}_{0}-\bar{R}_{c o m}^{\prime}\left(\mathbf{W}_{p}+\mathbf{Z}_{I T}\right)\right] \mathbf{h}_{k}-\bar{R}_{c o m}^{\prime} \mathbf{g}_{k}^{H} \mathbf{Z}_{P S} \mathbf{g}_{k} \\
\quad-\bar{R}_{c o m}^{\prime} \sigma_{k}^{2} \geq 0, \forall k \in \mathcal{K} \\
\text { (5a), (5b), (6a), (6b), (5e). }
\end{gathered}
$$

where $\alpha$ is a slack variable which can be interpreted as an upper bound on the eavesdropping rate constraints (8a) and (8b). Since problem (8) is still intractable, we treat it as a twolevel optimization problem where the inner and outer levels are defined, respectively, as follows

1) Inner level: Since $\log _{2}(\cdot)$ is monotonically increasing, problem (8) can be equivalently recast as the following inner level problem for given $\alpha \geq 1$,

$$
\begin{gathered}
g^{\text {opt }}\left(\bar{R}_{c o m}^{\prime}, \alpha\right)=\max _{\substack{\mathbf{V}_{\mathbf{Z}_{P S},}, \mathbf{W}_{0} \\
\mathbf{W}_{p}, \mathbf{Z}_{I T}}} \frac{\mathbf{h}_{1}^{H}\left(\mathbf{W}_{p}+\mathbf{Z}_{I T}\right) \mathbf{h}_{1}+\mathbf{g}_{1}^{H} \mathbf{Z}_{P S} \mathbf{g}_{1}+\sigma_{1}^{2}}{\alpha\left(\mathbf{h}_{1}^{H} \mathbf{Z}_{I T} \mathbf{h}_{1}+\mathbf{g}_{1}^{H} \mathbf{Z}_{P S} \mathbf{g}_{1}+\sigma_{1}^{2}\right)} \\
\text { s.t. (8a), (8b), (8c), (5a), (5b), (6a), (6b), (5e), }
\end{gathered}
$$


where $\bar{R}_{c o m}^{\prime}=2^{\bar{R}_{c o m}}-1$.

2) Outer level:

$$
\tilde{g}^{\mathrm{opt}}\left(\bar{R}_{c o m}^{\prime}\right)=\max _{\alpha \in\left[\alpha_{\min }, \alpha_{\max }\right]} g^{\mathrm{opt}}\left(\bar{R}_{c o m}^{\prime}, \alpha\right)
$$

where $\alpha_{\min }$ and $\alpha_{\max }$ are lower and upper bounds on the variable $\alpha$ determined as $\alpha_{\min }=1 \leq 1+$ $\frac{\mathbf{h}_{1}^{H} \mathbf{W}_{p} \mathbf{h}_{1}}{\mathbf{h}_{1} \mathbf{Z}_{I T} \mathbf{h}_{1}+\mathbf{g}_{1}^{H} \mathbf{Z}_{P S} \mathbf{g}_{1}+\sigma_{1}^{2}} \leq 1+\frac{\mathbf{h}_{1}^{H} \mathbf{W}_{p} \mathbf{h}_{1}}{\sigma_{1}^{2}}=1+\frac{\left\|\mathbf{h}_{1}\right\|^{2} \sum_{m=1}^{N_{I T}} p_{I T}^{m}}{\sigma_{1}^{2}}=$ $\alpha_{\max }$. Since (10) is a single-variable optimization problem, its optimal solution can be found via one-dimension search over $\alpha$.

With the optimal values $g^{\text {opt }}\left(\bar{R}_{c o m}^{\prime}, \alpha\right)$ and $\tilde{g}^{\text {opt }}\left(\bar{R}_{c o m}^{\prime}\right)$ of problems (9), and (10), respectively, the optimal value of problem (8) can be computed as $f^{\text {opt }}\left(\bar{R}_{c o m}^{\prime}\right)=\log _{2}\left(\tilde{g}^{\text {opt }}\left(\bar{R}_{c o m}^{\prime}\right)\right)$. The inner problem (9) is still nonconvex due to the convexity of its objective function as well as the constraints (8a) and (8b). To circumvent this issue, we first reformulate the constraints (8a) and (8b) as

$$
\begin{gathered}
(8 \mathrm{a}) \Rightarrow \mathbf{h}_{k}^{H}\left[\mathbf{W}_{p}-(\alpha-1) \mathbf{Z}_{I T}\right] \mathbf{h}_{k}-(\alpha-1) \mathbf{g}_{k}^{H} \mathbf{Z}_{P S} \mathbf{g}_{k} \\
\quad-(\alpha-1) \sigma_{k}^{2} \leq 0, k \in \overline{\mathcal{K}} \\
(8 \mathrm{~b}) \Rightarrow(\alpha-1)\left(\sigma_{e}^{2} \mathbf{I}+\mathbf{H}_{e}^{H} \mathbf{Z}_{I T} \mathbf{H}_{e}+\mathbf{G}_{e}^{H} \mathbf{Z}_{P S} \mathbf{G}_{e}\right) \\
\quad \\
\quad \mathbf{H}_{e}^{H} \mathbf{W}_{p} \mathbf{H}_{e} .
\end{gathered}
$$

Note that the constraints (8b) and (11b) are equivalent when $\alpha>1$ and $\operatorname{rank}\left(\mathbf{W}_{p}\right) \leq 1$ [11]. Thus, problem (9) can be equivalently modified as

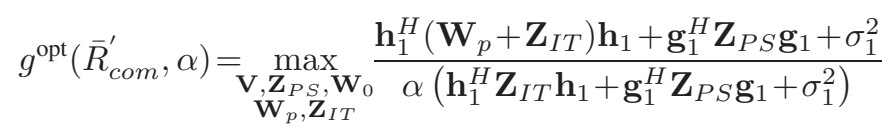

$$
\text { s.t. (11a), (11b), (8c), (5a), (5b), (6a), (6b), (5e). (12b) }
$$

Note that problem (12) is a quasi-convex problem, where its globally optimal solution can be found by the bi-section search method [41]. However, such a method will require solving a sequence of SDPs. Therefore, it is preferred to solve (12) by reformulating it as a convex problem. Here we explore a more efficient approach by applying the following Chanres-Cooper transformation [44], of the transmit covariance matrices,

$$
\mathbf{V}=\frac{\tilde{\mathbf{V}}}{\tau}, \mathbf{Z}_{P S}=\frac{\tilde{\mathbf{Z}}_{P S}}{\tau}, \mathbf{W}_{0}=\frac{\tilde{\mathbf{W}}_{0}}{\tau}, \mathbf{W}_{p}=\frac{\tilde{\mathbf{W}}_{p}}{\tau}, \mathbf{Z}_{I T}=\frac{\tilde{\mathbf{Z}}_{I T}}{\tau}, \tau>0 .
$$

Thus, problem (12) can be reformulated as the following SDP problem,

$$
\begin{aligned}
& g^{\mathrm{opt}}\left(\bar{R}_{c o m}^{\prime}, \alpha\right)=\max _{\substack{\tilde{\mathbf{V}}, \tilde{\mathbf{Z}}_{P S}, \tilde{\mathbf{W}}_{0} \\
\tilde{\mathbf{W}} p, \tilde{\mathbf{Z}}_{I T}, \tau}} \mathbf{h}_{1}^{H}\left(\tilde{\mathbf{W}}_{p}+\tilde{\mathbf{Z}}_{I T}\right) \mathbf{h}_{1}+\mathbf{g}_{1}^{H} \tilde{\mathbf{Z}}_{P S} \mathbf{g}_{1}+\sigma_{1}^{2} \tau \\
& \text { s.t. } \alpha\left(\tau \sigma_{1}^{2}+\mathbf{h}_{1}^{H} \tilde{\mathbf{Z}}_{I T} \mathbf{h}_{1}+\mathbf{g}_{1}^{H} \tilde{\mathbf{Z}}_{P S} \mathbf{g}_{1}\right)=1 \text {, } \\
& \mathbf{h}_{k}^{H}\left[\tilde{\mathbf{W}}_{p}-(\alpha-1) \tilde{\mathbf{Z}}_{I T}\right] \mathbf{h}_{k}-(\alpha-1) \mathbf{g}_{k}^{H} \tilde{\mathbf{Z}}_{P S} \mathbf{g}_{k} \\
& -(\alpha-1) \sigma_{k}^{2} \tau \leq 0, k \in \overline{\mathcal{K}}, \\
& (\alpha-1)\left(\sigma_{e}^{2} \tau \mathbf{I}+\mathbf{H}_{e}^{H} \tilde{\mathbf{Z}}_{I T} \mathbf{H}_{e}+\mathbf{G}_{e}^{H} \tilde{\mathbf{Z}}_{P S} \mathbf{G}_{e}\right) \succeq \mathbf{H}_{e}^{H} \tilde{\mathbf{W}}_{p} \mathbf{H}_{e}, \\
& \mathbf{h}_{k}^{H}\left[\tilde{\mathbf{W}}_{0}-\bar{R}_{c o m}^{\prime}\left(\tilde{\mathbf{W}}_{p}+\tilde{\mathbf{Z}}_{I T}\right)\right] \mathbf{h}_{k}-\bar{R}_{c o m}^{\prime} \mathbf{g}_{k}^{H} \tilde{\mathbf{Z}}_{P S} \mathbf{g}_{k} \\
& -\bar{R}_{c o m}^{\prime} \sigma_{k}^{2} \tau \geq 0 \\
& \operatorname{Tr}\left(\tilde{\mathbf{W}}_{0}+\tilde{\mathbf{W}}_{p}+\tilde{\mathbf{Z}}_{I T}\right) \leq \tau P_{I T} \\
& +\xi\left[\mathbf{h}^{H}\left(\tilde{\mathbf{V}}+\tilde{\mathbf{Z}}_{P S}\right) \mathbf{h}+\mathbf{h}_{S I}^{H}\left(\tilde{\mathbf{W}}_{0}+\tilde{\mathbf{W}}_{p}+\tilde{\mathbf{Z}}_{I T}\right) \mathbf{h}_{S I}\right], \\
& \operatorname{Tr}\left(\tilde{\mathbf{V}}+\tilde{\mathbf{Z}}_{P S}\right) \leq \tau P_{P S}, \tilde{\mathbf{V}}, \tilde{\mathbf{Z}}_{P S}, \tilde{\mathbf{W}}_{0}, \tilde{\mathbf{W}}_{p}, \tilde{\mathbf{Z}}_{I T} \succeq \mathbf{0}, \tau>0 . \\
& \operatorname{Tr}\left[\boldsymbol{\Xi}_{n}\left(\tilde{\mathbf{V}}+\tilde{\mathbf{Z}}_{P S}\right)\right] \leq \tau p_{P S}^{n}, \operatorname{Tr}\left[\boldsymbol{\Lambda}_{m}\left(\tilde{\mathbf{W}}_{0}+\tilde{\mathbf{W}}_{p}+\tilde{\mathbf{Z}}_{I T}\right)\right] \leq \tau p_{I T}^{m} .
\end{aligned}
$$

Note that the above transformation is motivated by the fact that the fractional objective function in (12a) is transformed to the linear objective function in (14a). In addition, this transformation fixes the denominator of (12a) which leads to the constraint in (14b). The proof of the equivalence of problems (12) and (14) can be obtained by following the argument in [10]. It is easily observed that (14) is an SDP, which can be efficiently solved to achieve the global optimization solution by using a convex optimization solver, such as CVX [45]. By exploiting problem (14), the optimal solution of (12) can be found through the relation (13) and its optimal value can be achieved for a given $\alpha$. Then, we adopt a one-dimensional search over $\alpha$ to solve the outer-level problem in (10), where the golden search can be employed to find the optimal $\alpha$ to achieve the maximum $g^{\text {opt }}\left(\bar{R}_{c o m}^{\prime}\right)$. The optimal value of problem $(8)$ can be computed as $f^{\text {opt }}\left(\bar{R}_{\text {com }}^{\prime}\right)=\log _{2}\left(\tilde{g}^{\text {opt }}\left(\bar{R}_{\text {com }}^{\prime}\right)\right)$ such that all Pareto optimal points of problem (5) are achieved. Note that our work can be extended to the multiple external eavesdroppers scenario, and the same algorithm can be applied to solve the SMRRM problem. These details are omitted to conserve space.

\section{B. Rank Revelation Analysis}

In the previous section, we adopted the SDP reformulation to solve the SMRRM problem (6). However, by solving its reformulated problem (12) numerically, there is generally no guarantee that the optimal solution of (14) would satisfy the rank-one condition for multicast service (i.e., $\mathbf{W}_{0}$ ) and the $\mathrm{AN}$ matrices (i.e., $\mathbf{Z}_{P S}$ and $\mathbf{Z}_{I T}$ ). However, the rank-one optimal solution for the confidential service (i.e., $\mathbf{W}_{p}$ ) and the energy beamforming matrix (i.e., V) can be guaranteed. Hence, in this subsection, we will exploit the structure of the optimal solution in problem (12). First, we denote the optimal objective value of (12) as $\bar{g}\left(\bar{R}_{\text {com }}^{\prime}, \alpha\right)$ for any given feasible $\alpha$. It can be verified that (12) has an identical optimal solution to the 
following power minimization problem [3], [11], [31], which is given by

$$
\begin{aligned}
& \quad \min _{\substack{\mathbf{V}, \mathbf{Z}_{P S} \\
\mathbf{W}_{0}, \mathbf{W}_{p}, \mathbf{Z}_{I T}}} \operatorname{Tr}\left(\mathbf{W}_{0}+\mathbf{W}_{p}+\mathbf{Z}_{I T}\right), \\
& \text { s.t. }(11 \mathrm{a}),(11 \mathrm{~b}),(8 \mathrm{c}),(5 \mathrm{a}),(5 \mathrm{~b}),(6 \mathrm{a}),(6 \mathrm{~b}),(5 \mathrm{e}), \\
& \frac{\mathbf{h}_{1}^{H}\left(\mathbf{W}_{p}+\mathbf{Z}_{I T}\right) \mathbf{h}_{1}+\mathbf{g}_{1}^{H} \mathbf{Z}_{P S} \mathbf{g}_{1}+\sigma_{1}^{2}}{\alpha\left(\mathbf{h}_{1}^{H} \mathbf{Z}_{I T} \mathbf{h}_{1}+\mathbf{g}_{1}^{H} \mathbf{Z}_{P S} \mathbf{g}_{1}+\sigma_{1}^{2}\right)} \geq \bar{g}^{\mathrm{opt}}\left(\bar{R}_{c o m}^{\prime}, \alpha\right) .
\end{aligned}
$$

Thus, the remaining part is to characterize the rank profile of problem (15). According to (15b), we have

$$
\mathbf{h}_{1}^{H}\left[\mathbf{W}_{p}+\alpha_{1} \mathbf{Z}_{I T}\right] \mathbf{h}_{1}-\alpha_{1} \mathbf{g}_{1}^{H} \mathbf{Z}_{P S} \mathbf{g}_{1}-\alpha_{1} \sigma_{1}^{2} \geq 0,
$$

where $\alpha_{1}=1-\alpha \bar{g}\left(\bar{R}_{c o m}^{\prime}, \alpha\right)$. Next, the Lagrange dual function of (15) can be expressed as (17) on the top of next page, where $\boldsymbol{\Omega}=$ $\left\{\mathbf{V}, \mathbf{Z}_{P S}, \mathbf{W}_{0}, \mathbf{W}_{p}, \mathbf{Z}_{I T}, \lambda_{k}, \mu_{k}, \delta, \gamma, \varrho_{n}, \varphi_{m}, \mathbf{A}, \mathbf{B}, \mathbf{C}, \mathbf{D}, \mathbf{F}, \mathbf{X}\right.$ $\mathbf{A} \succeq \mathbf{0}, \mathbf{B} \succeq \mathbf{0}, \mathbf{C} \succeq \mathbf{0}, \mathbf{D} \succeq \mathbf{0}, \mathbf{F} \succeq \mathbf{0}, \mathbf{X} \succeq \mathbf{0}, \lambda_{k} \geq 0$, $\mu_{k} \geq 0, \delta \geq 0, \gamma \geq 0, \varrho_{n} \geq 0$, and $\varphi_{m} \geq 0$ are dual variables pertaining to the primary constraints in (15).

Theorem 2: Provided that the optimization problem (12) is feasible for any given $\alpha$, the optimal solution of the confidential service (i.e., $\left.\mathbf{W}_{p}\right)$ satisfies $\operatorname{rank}\left(\mathbf{W}_{p}\right) \leq 1$ when $\mathbf{I}-\xi \mathbf{h}_{S I} \mathbf{S I}^{H} \succeq \mathbf{0}$ holds. Moreover, provided that the energy beamforming matrix condition $\mathbf{V} \neq \mathbf{0}$ holds, it always yields a rank-one solution, i.e., $\operatorname{rank}(\mathbf{V}) \leq 1$.

Proof: Please refer to Appendix A.

Now, a natural question arises on how to tackle a more general scenario where the optimal solution of problem (15), denoted as $\left(\mathbf{V}^{*}, \mathbf{Z}_{P S}^{*}, \mathbf{W}_{0}^{*}, \mathbf{W}_{p}^{*}, \mathbf{Z}_{I T}^{*}\right)$, yields $\operatorname{rank}\left(\mathbf{W}_{p}^{*}\right) \geq 1$. For this scenario, we construct a new solution set for problem (15) with the optimal solution of the confidential service.

Theorem 3: Suppose that the optimal solution of problem (15) is denoted as $\left(\mathbf{V}^{*}, \mathbf{Z}_{P S}^{*}, \mathbf{W}_{0}^{*}, \mathbf{W}_{p}^{*}, \mathbf{Z}_{I T}^{*}\right)$ such that $\operatorname{rank}\left(\mathbf{W}_{p}\right)>1$. Then, there exists a feasible solution of (15), denoted as $\left(\tilde{\mathbf{V}}^{*}, \tilde{\mathbf{Z}}_{P S}^{*}, \tilde{\mathbf{W}}_{0}^{*}, \tilde{\mathbf{W}}_{p}^{*}, \tilde{\mathbf{Z}}_{I T}^{*}\right)$, which achieves the same objective value with $\left(\mathbf{V}^{*}, \mathbf{Z}_{P S}^{*}, \mathbf{W}_{0}^{*}, \mathbf{W}_{p}^{*}, \mathbf{Z}_{I T}^{*}\right)$ with $\operatorname{rank}\left(\tilde{\mathbf{W}}_{p}^{*}\right)=1$.

Proof: Please refer to Appendix B.

\section{The Worst-CAse SMRRM Problem BAsed on IMPERFECT CSI}

In the previous section, we solved the SMRRM problem based on the assumption that the CSIs are perfectly available at the PS and IT. However, there are practical challenges in obtaining the CSIs due to the channel estimation mismatch and channel quantization errors [3], [12]. Hence, in this section, we investigate the worst-case SMRRM problem based on the imperfect CSI case. By incorporating the channel uncertainties, this optimization framework can be reformulated into an SDP.

\section{A. Channel Uncertainty Model}

In this subsection, we consider a more general scenario where the PS and FD IT have imperfect CSI of the links between the IT and all users, the PS and all users, the FD IT and the eavesdropper, as well as the PS and the eavesdropper, respectively. We express the actual channels of these links as the sum of their associated estimates plus the channel estimation errors, i.e.,

$$
\begin{aligned}
& \mathcal{S}_{1, k}=\left\{\mathcal{S}_{1, k} \mid \mathbf{h}_{k}=\overline{\mathbf{h}}_{k}+\mathbf{e}_{1, k}, \mathbf{e}_{1, k}^{H} \mathbf{e}_{1, k} \leq \varepsilon_{1, k}^{2}, \forall k \in \mathcal{K}\right\}, \\
& \mathcal{S}_{2, k}=\left\{\mathcal{S}_{2, k} \mid \mathbf{g}_{k}=\overline{\mathbf{g}}_{k}+\mathbf{e}_{2, k}, \mathbf{e}_{2, k}^{H} \mathbf{e}_{2, k} \leq \varepsilon_{2, k}^{2}, \forall k \in \mathcal{K}\right\}, \\
& \mathcal{S}_{e, 1}=\left\{\mathcal{S}_{e, 1} \mid \mathbf{H}_{e}=\overline{\mathbf{H}}_{e}+\mathbf{E}_{1}, \operatorname{Tr}\left(\mathbf{E}_{1} \mathbf{E}_{1}^{H}\right) \leq \varepsilon_{e, 1}^{2}\right\}, \\
& \mathcal{S}_{e, 2}=\left\{\mathcal{S}_{e, 2} \mid \mathbf{G}_{e}=\overline{\mathbf{G}}_{e}+\mathbf{E}_{2}, \operatorname{Tr}\left(\mathbf{E}_{2} \mathbf{E}_{2}^{H}\right) \leq \varepsilon_{e, 2}^{2}\right\},
\end{aligned}
$$

where $\overline{\mathbf{h}}_{k}, \overline{\mathbf{g}}_{k}, \mathbf{H}_{e}$, and $\mathbf{G}_{e}$ are the estimated versions of $\mathbf{h}_{k}$, $\mathbf{g}_{k}, \mathbf{H}_{e}$, and $\mathbf{G}_{e}$, respectively, while, $\mathbf{e}_{1, k}, \mathbf{e}_{2, k}, \mathbf{E}_{1}$, and $\mathbf{E}_{2}$ represent the associated channel estimation errors which are based on the ellipsoidal channel error models [11]-[13], [31].

\section{B. The Worst-Case Robust SMRRM}

According to the channel uncertainty model in Section IV-A, the worst-case secrecy rate region can be expressed as ffollows

$\bar{R}_{c o m, w c} \leq \min _{k \in \mathcal{K}} \min _{\substack{\mathbf{h}_{k} \in \mathcal{S}_{1, k} \\ \mathbf{g}_{k} \in \mathcal{S}_{2, k}}} R_{c o m, k}=R_{c o m, w c}$,
$\bar{R}_{s e c, w c} \leq \min _{\substack{\mathbf{h}_{1} \in \mathcal{S}_{1,1} \\ \mathbf{g}_{1} \in \mathcal{S}_{2,1}}} R_{1}-\max \left\{\max _{k \in \mathcal{\mathcal { K }} \mathbf{h}_{k} \in \mathcal{S}_{1, k}} \max _{\mathbf{g}_{k} \in \mathcal{S}_{2, k}} R_{k}, \max _{\substack{\mathbf{H}_{e} \in \mathcal{S}_{e, 1} \\ \mathbf{g}_{k} \in \mathcal{S}_{e, 2}}} R_{e}\right\}=R_{s e c, w c}$

To develop robust designs for the energy matrix $\mathbf{V}$, the multicast beamforming matrix $\mathbf{W}_{0}$, the confidential matrix $\mathbf{W}_{p}$, and the AN matrices $\left(\mathbf{Z}_{P S}\right.$ and $\left.\mathbf{Z}_{I T}\right)$, we formulate the following worst-case SMRRM problem,

$$
\begin{aligned}
& \underset{\substack{\mathbf{V}, \mathbf{Z}_{P S}, \mathbf{W}_{0} \\
\mathbf{w}_{p}, \mathbf{Z}_{I T}, \bar{R}_{c o m}, \bar{R}_{s e c}}}{\bigcup_{\mathbb{R}_{+}^{2}}}\left(\bar{R}_{c o m, w c}, \bar{R}_{s e c, w c}\right), \\
& \text { s.t. (19a), (19b), (5a), (5b), (6a), (6b), (5e). }
\end{aligned}
$$

Problem (20) is not convex due to channel uncertainties, which cannot be solved directly. To circumvent this issue, we recast (20) into a standard scalar optimization problem similar to the procedure shown in Section III. By fixing the variable $\bar{R}_{c o m, w c} \geq 0$, we modify problem (20) as follows

$$
\begin{aligned}
f_{w c}^{\mathrm{opt}}\left(\bar{R}_{c o m, w c}\right)= & \max _{\substack{\mathbf{V}, \mathbf{Z}_{P S} \\
\mathbf{W}_{0}, \mathbf{W}_{p}, \mathbf{Z}_{I T}}} R_{s e c, w c}, \\
& \text { s.t. (19a), (5a), (5b), (6a), (6b), (5e). }
\end{aligned}
$$

From (21), $\bar{R}_{c o m, w c}$ is a pre-defined minimum achievable worst-case multicast rate, and when $\bar{R}_{\text {com }}=0$, (21) is a conventional worst-case SRM problem for a secure wireless powered FD energy recycle system. Similar to (7), the maximum value of $\bar{R}_{c o m, w c}$, denoted by $\bar{R}_{c o m, w c}^{\max }$, is attained by solving the following problem

$$
\bar{R}_{c o m, w c}^{\max }=\max _{\mathbf{W}_{0} \succeq \mathbf{0}} \min _{\mathbf{h}_{k} \in \mathcal{S}_{1, k}} \log _{2}\left(1+\frac{\mathbf{h}_{k}^{H} \mathbf{W}_{0} \mathbf{h}_{k}}{\sigma_{k}^{2}}\right) \text {, s.t. (7b), }
$$

where $\bar{R}_{c o m, w c}^{\max }$ is the largest achievable worst-case multicast rate. Problem (22) can be easily reformulated into an SDP and can be solved by using CVX [45]. Note that the rate pair $\left(\bar{R}_{c o m, w c}, f_{w c}^{\text {opt }}\left(\bar{R}_{c o m}\right)\right)$ is a Pareto optimal point of the problem (21), and all Pareto optimal points of (21) can be achieved with $\bar{R}_{c o m, w c} \in\left[0, \bar{R}_{c o m, w c}^{\max }\right]$. 


$$
\begin{aligned}
& \mathcal{L}(\boldsymbol{\Omega})=\operatorname{Tr}\left(\mathbf{W}_{0}\right)+\operatorname{Tr}\left(\mathbf{W}_{p}\right)+\operatorname{Tr}\left(\mathbf{Z}_{I T}\right)-\operatorname{Tr}(\mathbf{A V})-\operatorname{Tr}\left(\mathbf{B} \mathbf{Z}_{P S}\right)-\operatorname{Tr}\left(\mathbf{C} \mathbf{W}_{0}\right)-\operatorname{Tr}\left(\mathbf{D} \mathbf{W}_{p}\right)-\operatorname{Tr}\left(\mathbf{F} \mathbf{Z}_{I T}\right) \\
& -\lambda_{1}\left[\mathbf{h}_{1}^{H}\left[\mathbf{W}_{p}+\alpha_{1} \mathbf{Z}_{I T}\right] \mathbf{h}_{1}-\alpha_{1} \mathbf{g}_{1}^{H} \mathbf{Z}_{P S} \mathbf{g}_{1}-\alpha_{1} \sigma_{1}^{2}\right]+\sum_{k=2}^{K} \lambda_{k}\left[\mathbf{h}_{k}^{H}\left[\mathbf{W}_{p}-(\alpha-1) \mathbf{Z}_{I T}\right] \mathbf{h}_{k}-(\alpha-1) \mathbf{g}_{k}^{H} \mathbf{Z}_{P S} \mathbf{g}_{k}\right. \\
& \left.-(\alpha-1) \sigma_{k}^{2}\right]-\operatorname{Tr}\left[\mathbf{X}\left((\alpha-1)\left(\sigma_{e}^{2} \mathbf{I}+\mathbf{H}_{e}^{H} \mathbf{Z}_{I T} \mathbf{H}_{e}+\mathbf{G}_{e}^{H} \mathbf{Z}_{P S} \mathbf{G}_{e}\right)-\mathbf{H}_{e}^{H} \mathbf{W}_{p} \mathbf{H}_{e}\right)\right]-\sum_{k=1}^{K} \mu_{k}\left[\mathbf { h } _ { k } ^ { H } \left[\mathbf{W}_{0}\right.\right. \\
& \left.\left.-\bar{R}_{c o m}^{\prime}\left(\mathbf{W}_{p}+\mathbf{Z}_{I T}\right)\right] \mathbf{h}_{k}-\bar{R}_{c o m}^{\prime} \mathbf{g}_{k}^{H} \mathbf{Z}_{P S} \mathbf{g}_{k}-\bar{R}_{c o m}^{\prime} \sigma_{k}^{2}\right]+\delta\left[\operatorname{Tr}\left[\left(\mathbf{I}-\xi \mathbf{h}_{S I} \mathbf{h}_{S I}^{H}\right)\left(\mathbf{W}_{0}+\mathbf{W}_{p}+\mathbf{Z}_{I T}\right)\right]\right. \\
& \left.-P_{I T}-\xi \operatorname{Tr}\left[\mathbf{h h}^{H}\left(\mathbf{V}+\mathbf{Z}_{P S}\right)\right]\right]+\gamma\left[\operatorname{Tr}\left(\mathbf{V}+\mathbf{Z}_{P S}\right)-P_{P S}\right]+\sum_{n=1}^{N_{P S}} \varrho_{n}\left[\operatorname{Tr}\left[\mathbf{\Xi}_{n}\left(\mathbf{V}+\mathbf{Z}_{P S}\right)\right]-p_{P S}^{n}\right] \\
& +\sum_{m=1}^{N_{I T}} \varphi_{m}\left[\operatorname{Tr}\left[\mathbf{\Lambda}_{m}\left(\mathbf{W}_{0}+\mathbf{W}_{p}+\mathbf{Z}_{I T}\right)\right]-p_{I T}^{m}\right]
\end{aligned}
$$

Next, we develop a tractable reformulation of problem (21). Similar to Section III, we employ a slack variable $\alpha_{2}$ such that (21) can be modified as (23) on the top of next page, where $\bar{R}_{c o m, w c}^{\prime}=2^{\bar{R}_{c o m, w c}}-1$. Problem (23) is still nonconvex, and challenging to solve. Similar to Section III, we follow a two-level optimization approach to solve (23). First, problem (23) is reformulated as a single-variable optimization problem over $\alpha_{2}$, and its optimal solution can be found via one-dimensional search (i.e., golden search) over the interval $\left[\alpha_{2, \min }, \alpha_{2, \max }\right]$. The lower and upper bounds, denoted by, $\alpha_{2, \min }$ and $\alpha_{2, \max }$, respectively, can be easily determined to be $\alpha_{2, \text { min }}=1 \leq \alpha_{2} \leq 1+\min _{\mathbf{h}_{1} \in \mathcal{S}_{1,1}} \frac{1}{\sigma_{1}^{2}} \mathbf{h}_{1}^{H} \mathbf{W}_{p} \mathbf{h}_{1} \leq 1+$ $\frac{1}{\sigma_{1}^{2}}\left(\left\|\overline{\mathbf{h}}_{1}\right\|-\varepsilon_{1,1}\right)^{2} \sum_{m=1}^{N_{I T}} p_{I T}^{m}=\alpha_{2, \max }$ Thus, the outer level problem is given by

$$
\begin{array}{r}
\tilde{g}_{w c}^{\mathrm{opt}}\left(\bar{R}_{c o m, w c}^{\prime}\right)=\max _{\alpha_{2}} g_{w c}^{\mathrm{opt}}\left(\bar{R}_{c o m, w c}^{\prime}, \alpha_{2}\right), \\
\text { s.t. } \alpha_{2, \min } \leq \alpha_{2} \leq \alpha_{2, \max },
\end{array}
$$

where $f_{w c}^{\mathrm{opt}}\left(\bar{R}_{c o m, w c}^{\prime}\right)=\log _{2} \tilde{g}^{\mathrm{opt}}\left(\bar{R}_{c o m, w c}^{\prime}\right), \quad$ and $g_{w c}^{\mathrm{opt}}\left(\bar{R}_{c o m, w c}^{\prime}, \alpha_{2}\right)$ can be written as

$$
\begin{aligned}
& \max _{\substack{\mathbf{V}, \mathbf{Z}_{P S} \\
\mathbf{W}_{0}, \mathbf{W}_{p}, \mathbf{Z}_{I T}}} \min _{\substack{\mathbf{h}_{1} \in \mathcal{S}_{1,1} \\
\mathbf{g}_{1,1}}} \frac{\mathbf{h}_{1}^{H}\left(\mathbf{W}_{p}+\mathbf{Z}_{I T}\right) \mathbf{h}_{1}+\mathbf{g}_{1}^{H} \mathbf{Z}_{P S} \mathbf{g}_{1}+\sigma_{1}^{2}}{\alpha_{2}\left(\mathbf{h}_{1}^{H} \mathbf{Z}_{I T} \mathbf{h}_{1}+\mathbf{g}_{1}^{H} \mathbf{Z}_{P S} \mathbf{g}_{1}+\sigma_{1}^{2}\right)} \\
& \text { s.t. (23a), (23b), (23c), (5a), (5b), (6a), (6b), (5e). }
\end{aligned}
$$

In the following, we reformulate problem (25) for a given $\alpha_{2}$. The challenge of dealing with (25) lies in the existence of channel uncertainties in the objective function (25a), as well as the constraints (23a), (23b), and (23c). Now, we first employ the S-Procedure in [41] and [46, Theorem 3.5] such that the constraints (23c) and (23a) can be reformulated into the linear matrix inequalities (LMI), i.e., (26a) and (26b) on the top of next page, respectively, where $\left\{\bar{\lambda}_{k}, \bar{\gamma}_{k}, \bar{\beta}_{k}, \bar{\theta}_{k} \geq 0\right\}$ are the auxiliary optimization variables, $s_{k}=\overline{\mathbf{h}}_{k}^{H} \overline{\mathbf{A}}_{1} \overline{\mathbf{h}}_{k}$ $\overline{\mathbf{g}}_{k}^{H} \overline{\mathbf{A}}_{2} \overline{\mathbf{g}}_{k}-\bar{R}_{c o m, w c}^{\prime} \sigma_{k}^{2}-\bar{\lambda}_{k} \varepsilon_{1, k}^{2}-\bar{\gamma}_{k}, \bar{R}_{c o m, w c}^{\prime}=2^{\bar{R}_{c o m, w c}-1}$, $\overline{\mathbf{A}}_{1}=\mathbf{W}_{0}-\bar{R}_{c o m, w c}^{\prime}\left(\mathbf{W}_{p}+\mathbf{Z}_{I T}\right), \overline{\mathbf{A}}_{2}=\bar{R}_{c o m, w c}^{\prime} \mathbf{Z}_{P S}, \overline{\mathbf{B}}_{1}=$ $\mathbf{W}_{p}-\left(\alpha_{2}-1\right) \mathbf{Z}_{I T}, \overline{\mathbf{B}}_{2}=\left(\alpha_{2}-1\right) \mathbf{Z}_{P S}$, and $t_{k}=-\overline{\mathbf{h}}_{k}^{H} \overline{\mathbf{B}}_{1} \overline{\mathbf{h}}_{k}+$ $\overline{\mathbf{g}}_{k}^{H} \overline{\mathbf{B}}_{2} \overline{\mathbf{g}}_{k}+\left(\alpha_{2}-1\right) \sigma_{k}^{2}-\bar{\beta}_{k} \varepsilon_{1, k}^{2}-\bar{\theta}_{k}$. To proceed, by exploiting [46, Theorem 3.3 and 3.5] the constraint (23b) is recast as (27) on the top of next page, where $t_{e}=\overline{\mathbf{H}}_{e}^{H} \overline{\mathbf{C}}_{1} \overline{\mathbf{H}}_{e}+\overline{\mathbf{G}}_{e}^{H} \overline{\mathbf{C}}_{2} \overline{\mathbf{G}}_{e}+$ $\left[\left(\alpha_{2}-1\right) \sigma_{e}^{2}-\bar{\delta}_{e}-\bar{\eta}_{e}\right] \mathbf{I}_{N_{E}}, \overline{\mathbf{C}}_{1}=\left(\alpha_{2}-1\right) \mathbf{Z}_{I T}-\mathbf{W}_{p}$, and $\mathbf{C}_{2}=\left(\alpha_{2}-1\right) \mathbf{Z}_{P S}$. Thus, problem (25) can be reformulated as

$$
\begin{aligned}
& \max _{\boldsymbol{\Omega}} \min _{\mathbf{h}_{1} \in \mathcal{S}_{1,1}} \frac{\mathbf{h}_{1}^{H}\left(\mathbf{W}_{p}+\mathbf{Z}_{I T}\right) \mathbf{h}_{1}+\mathbf{g}_{1}^{H} \mathbf{Z}_{P S} \mathbf{g}_{1}+\sigma_{1}^{2}}{\alpha_{2}\left(\mathbf{h}_{1}^{H} \mathbf{Z}_{I T} \mathbf{h}_{1}+\mathbf{g}_{1}^{H} \mathbf{Z}_{P S} \mathbf{g}_{1}+\sigma_{1}^{2}\right)} \\
& \text { s.t. (5a), (5b), (6a), (6b), (5e), (26a), (26b), (27), } \\
& \left\{\mathbf{V}, \mathbf{Z}_{P S}, \mathbf{W}_{0}, \mathbf{W}_{p}, \mathbf{Z}_{I T}, \bar{\lambda}_{k}, \bar{\gamma}_{k}, \bar{\beta}_{k}, \bar{\theta}_{k}, \bar{\delta}_{e}, \bar{\eta}_{e}\right\} \in \boldsymbol{\Omega} .
\end{aligned}
$$

With the optimal value of $g_{w c}^{\mathrm{opt}}\left(\bar{R}_{c o m, w c}^{\prime}, \alpha_{2}\right)$ of problem (28) computed, the optimal $f_{w c}^{\mathrm{opt}}\left(\bar{R}_{c o m, w c}^{\prime}\right)$ of problem (23) can be found via $\bar{f}_{w c}^{\mathrm{opt}}\left(\bar{R}_{c o m, w c}^{\prime}\right)=\log _{2}\left[\tilde{g}_{w c}^{\mathrm{opt}}\left(\bar{R}_{c o m, w c}^{\prime}\right)\right]$. To proceed, it is observed that problem (28) is still nonconvex. Thus, we will show that (28) is a quasi-convex optimization problem such that its globally optimal solution can be efficiently found via the bisection method [41].

Theorem 4: The problem (28) is a quasi-convex problem.

Proof: Please refer to Appendix C.

By applying Theorem 4, we adopt the bi-section method to search $\tilde{\alpha}$ to obtain the globally optimal solution of the problem (28). However, such a method would require solving a sequence of SDPs, which leads to a very high computational complexity. To reduce complexity, we consider a suboptimal resource allocation scheme to reformulate problem (28) in a more efficient manner by employing the CharnesCooper transformation. By introducing $\bar{\tau}>0$ and defining $\mathbf{V}=\frac{\hat{\mathbf{V}}}{\bar{\tau}}, \mathbf{Z}_{P S}=\frac{\hat{\mathbf{Z}}_{P S}}{\bar{\tau}}, \mathbf{W}_{0}=\frac{\hat{\mathbf{W}}_{0}}{\bar{\tau}}, \mathbf{W}_{p}=\frac{\hat{\mathbf{W}}_{p}}{\bar{\tau}}, \mathbf{Z}_{P S}=$ $\frac{\hat{\mathbf{Z}}_{P S}}{\bar{\tau}}, \bar{\lambda}_{k}=\frac{\hat{\lambda}_{k}}{\bar{\tau}}, \bar{\gamma}_{k}=\frac{\hat{\gamma}_{k}}{\bar{\tau}}, \forall k \in \mathcal{K}, \bar{\beta}_{k}=\frac{\hat{\beta}_{k}}{\bar{\tau}}, \bar{\theta}_{k}=$ $\frac{\hat{\gamma}_{k}}{\bar{\tau}}, \forall k \in \overline{\mathcal{K}}, \bar{\delta}_{e} \geq 0, \bar{\eta}_{e} \geq 0$, problem (28) can be reformulated as (29) shown on the top of next page, where $\hat{\mathbf{A}}_{1}=\hat{\mathbf{W}}_{0}-\bar{R}_{c o m, w c}^{\prime}\left(\hat{\mathbf{W}}_{p}+\hat{\mathbf{Z}}_{I T}\right), \hat{\mathbf{A}}_{2}=\bar{R}_{c o m, w c}^{\prime} \hat{\mathbf{Z}}_{I T}$, $\hat{\mathbf{B}}_{1}=\hat{\mathbf{W}}_{p}-\left(\alpha_{2}-1\right) \hat{\mathbf{Z}}_{I T}, \hat{\mathbf{B}}_{2}=\left(\alpha_{2}-1\right) \hat{\mathbf{Z}}_{P S}, \hat{\mathbf{C}}_{1}=$ $\left(\alpha_{2}-1\right) \hat{\mathbf{Z}}_{I T}-\hat{\mathbf{W}}_{p}, \hat{\mathbf{C}}_{2}=\left(\alpha_{2}-1\right) \hat{\mathbf{Z}}_{P S}, \mathbf{D}_{1}=\hat{\mathbf{W}}_{p}+\hat{\mathbf{Z}}_{I T}$, $s_{1}=\overline{\mathbf{h}}_{1}^{H} \mathbf{D}_{1} \overline{\mathbf{h}}_{1}+\overline{\mathbf{g}}_{1}^{H} \mathbf{Z}_{P S} \overline{\mathbf{g}}_{1}+\bar{\tau} \sigma_{1}^{2}-t-\hat{\beta}_{1} \varepsilon_{1,1}^{2}-\hat{\eta}_{1}$, and $s_{2}=-\overline{\mathbf{h}}_{1}^{H} \mathbf{Z}_{I T}-\overline{\mathbf{g}}_{1}^{H} \mathbf{Z}_{P S} \overline{\mathbf{g}}_{1}-\bar{\tau} \sigma_{1}^{2}+\frac{1}{\alpha_{2}}-\hat{\beta}_{11} \varepsilon_{1,1}^{2}-\hat{\eta}_{11}$. Problem (29) is a standard SDP, which can be efficiently solved by using the interior-point method [41]. It is observed that (29) can significantly reduce the computational complexity compared with the globally optimal solution. In addition, this 


$$
f_{w c}^{\text {opt }}\left(\bar{R}_{c o m, w c}^{\prime}\right)=\max _{\substack{\mathbf{V}, \mathbf{Z}_{P S}, \mathbf{W}_{0} \\ \mathbf{W}_{p}, \mathbf{Z}_{I T}, \alpha_{2}}} \min _{\mathbf{g}_{1} \in \mathcal{S}_{1,1} \in \mathcal{S}_{2,1}} \log _{2}\left(\frac{\mathbf{h}_{1}^{H}\left(\mathbf{W}_{p}+\mathbf{Z}_{I T}\right) \mathbf{h}_{1}+\mathbf{g}_{1}^{H} \mathbf{Z}_{P S} \mathbf{g}_{1}+\sigma_{1}^{2}}{\alpha_{2}\left(\mathbf{h}_{1}^{H} \mathbf{Z}_{I T} \mathbf{h}_{1}+\mathbf{g}_{1}^{H} \mathbf{Z}_{P S} \mathbf{g}_{1}+\sigma_{1}^{2}\right)}\right),
$$

s.t. (5a), (5b), (6a), (6b), (5e),

$$
\begin{aligned}
& \max _{k \in \mathcal{K}} \max _{\substack{\mathbf{h}_{k} \in \mathcal{S}_{1, k} \\
\mathbf{g}_{k} \in \mathcal{S}_{2, k}}} \log _{2}\left(1+\frac{\mathbf{h}_{k}^{H} \mathbf{W}_{p} \mathbf{h}_{k}}{\mathbf{h}_{k}^{H} \mathbf{Z}_{I T} \mathbf{h}_{k}+\mathbf{g}_{k}^{H} \mathbf{Z}_{P S} \mathbf{g}_{k}+\sigma_{k}^{2}}\right) \leq \log _{2}\left(\alpha_{2}\right), \\
& \max _{\substack{\mathbf{H}_{e} \in \mathcal{S}_{e, 1} \\
\mathbf{G}_{e} \in \mathcal{S}_{e, 2}}} \log _{2}\left|\mathbf{I}+\left(\sigma_{e}^{2} \mathbf{I}+\mathbf{H}_{e}^{H} \mathbf{Z}_{I T} \mathbf{H}_{e}+\mathbf{G}_{e}^{H} \mathbf{Z}_{P S} \mathbf{G}_{e}\right)^{-1} \mathbf{H}_{e}^{H} \mathbf{W}_{p} \mathbf{H}_{e}\right| \leq \log _{2}\left(\alpha_{2}\right), \\
& \mathbf{h}_{k}^{H}\left(\mathbf{W}_{0}-\bar{R}_{c o m, w c}^{\prime}\left(\mathbf{W}_{p}+\mathbf{Z}_{I T}\right)\right) \mathbf{h}_{k}-\bar{R}_{c o m, w}^{\prime} \mathbf{g}_{k}^{H} \mathbf{Z}_{P S} \mathbf{g}_{k}-\bar{R}_{c o m, w c}^{\prime} \sigma_{k}^{2} \geq 0 .
\end{aligned}
$$

$$
\begin{gathered}
\mathbf{S}_{k}\left(\mathbf{W}_{0}, \mathbf{W}_{p}, \mathbf{Z}_{I T}, \mathbf{Z}_{P S}, \bar{\lambda}_{k}, \bar{\gamma}_{k}\right)=\left[\begin{array}{ccc}
\bar{\lambda}_{k} \mathbf{I}_{N_{I T}}+\overline{\mathbf{A}}_{1} & \overline{\mathbf{A}}_{1} \overline{\mathbf{h}}_{k} & \mathbf{0}_{N_{I T} \times N_{P S}} \\
\overline{\mathbf{h}}_{k}^{H} \overline{\mathbf{A}}_{1} & s_{k} & -\overline{\mathbf{g}}_{k}^{H} \overline{\mathbf{A}}_{2} \\
\mathbf{0}_{N_{P S} \times N_{I T}} & -\overline{\mathbf{A}}_{2} \overline{\mathbf{g}}_{k} & -\overline{\mathbf{A}}_{2}+\frac{\bar{\gamma}_{k}}{\varepsilon_{2, k}^{2}} \mathbf{\mathbf { I }}_{N_{P S}}
\end{array}\right] \succeq \mathbf{0}, \forall k \in \mathcal{K} . \\
\mathbf{T}_{k}\left(\mathbf{W}_{p}, \mathbf{Z}_{I T}, \mathbf{Z}_{P S}, \bar{\beta}_{k}, \bar{\theta}_{k}\right)=\left[\begin{array}{ccc}
\bar{\beta}_{k} \mathbf{I}_{N_{I T}}-\overline{\mathbf{B}}_{1} & -\overline{\mathbf{B}}_{1} \overline{\mathbf{g}}_{k} & \mathbf{0}_{N_{I T} \times N_{P S}} \\
-\overline{\mathbf{h}}_{k}^{H} \overline{\mathbf{B}}_{1} & t_{k} & \overline{\mathbf{g}}_{k}^{H} \overline{\mathbf{B}}_{2} \\
\mathbf{0}_{N_{P S} \times N_{I T}} & \overline{\mathbf{B}}_{2} \overline{\mathbf{g}}_{k} & \overline{\mathbf{B}}_{2}+\frac{\bar{\theta}_{k}}{\varepsilon_{2, k}^{2}} \mathbf{I}_{N_{P S}}
\end{array}\right] \succeq \mathbf{0}, \forall k \in \overline{\mathcal{K}},
\end{gathered}
$$

$$
\mathbf{T}_{e}\left(\mathbf{W}_{p}, \mathbf{Z}_{I T}, \mathbf{Z}_{P S}, \bar{\delta}_{e}, \bar{\eta}_{e}\right)=\left[\begin{array}{ccc}
\frac{\bar{\delta}_{e}}{\varepsilon_{e, 1}^{2}} \mathbf{I}_{N_{I T}}+\overline{\mathbf{C}}_{1} \overline{\mathbf{C}}_{1} \overline{\mathbf{H}}_{e} & \mathbf{0}_{N_{I T} \times N_{P S}} \\
\overline{\mathbf{H}}_{e}^{H} \overline{\mathbf{C}}_{1} & t_{e} & \overline{\mathbf{G}}_{e}^{H} \overline{\mathbf{C}}_{2} \\
\mathbf{0}_{N_{P S} \times N_{T}} & \overline{\mathbf{C}}_{2} \overline{\mathbf{G}}_{e} & \overline{\mathbf{C}}_{2}+\frac{\bar{\eta}_{e}}{\varepsilon_{2, e}^{2}} \mathbf{I}_{N_{P S}}
\end{array}\right] \succeq \mathbf{0}, \bar{\delta}_{e} \geq 0, \bar{\eta}_{e} \geq 0 .
$$

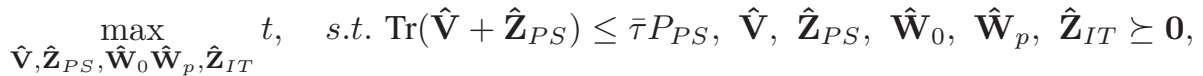

$$
\begin{aligned}
& \hat{\lambda}_{k}, \hat{\gamma}_{k}, \hat{\beta}_{k}, \hat{\theta}_{k}, \hat{\delta}_{e}, \hat{\eta}_{e}, \bar{\tau} \\
& \operatorname{Tr}\left(\hat{\mathbf{W}}_{0}+\hat{\mathbf{W}}_{p}+\hat{\mathbf{Z}}_{I T}\right) \leq \bar{\tau} P_{I T}+\xi\left[\mathbf{h}^{H}\left(\hat{\mathbf{V}}+\hat{\mathbf{Z}}_{P S}\right) \mathbf{h}+\mathbf{h}_{S I}^{H}\left(\hat{\mathbf{W}}_{0}+\hat{\mathbf{W}}_{p}+\hat{\mathbf{Z}}_{I T}\right) \mathbf{h}_{S I}\right], \\
& \operatorname{Tr}\left[\boldsymbol{\Xi}_{n}\left(\hat{\mathbf{V}}+\hat{\mathbf{Z}}_{P S}\right)\right] \leq \bar{\tau} p_{P S}^{n}, \operatorname{Tr}\left[\boldsymbol{\Lambda}_{m}\left(\hat{\mathbf{W}}_{0}+\hat{\mathbf{W}}_{p}+\hat{\mathbf{Z}}_{I T}\right)\right] \leq \bar{\tau} p_{I T}^{m},
\end{aligned}
$$

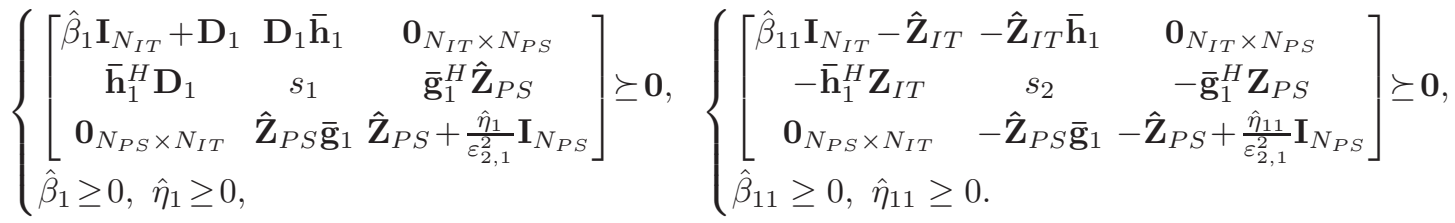

$$
\begin{aligned}
& \left\{\begin{array} { l } 
{ \mathbf { S } _ { k } ( \hat { \mathbf { W } } _ { 0 } , \hat { \mathbf { W } } _ { p } , \hat { \mathbf { Z } } _ { I T } , \hat { \mathbf { Z } } _ { P S } , \hat { \lambda } _ { k } , \hat { \gamma } _ { k } ) \succeq \mathbf { 0 } , } \\
{ \hat { \lambda } _ { k } \geq 0 , \hat { \gamma } _ { k } \geq 0 , \forall k \in \mathcal { K } , }
\end{array} \left\{\begin{array}{l}
\mathbf{T}_{k}\left(\hat{\mathbf{W}}_{p}, \hat{\mathbf{Z}}_{I T}, \hat{\mathbf{Z}}_{P S}, \hat{\beta}_{k}, \hat{\theta}_{k}\right) \succeq \mathbf{0}, \\
\hat{\beta}_{k} \geq 0, \hat{\theta}_{k} \geq 0, \forall k \in \overline{\mathcal{K}},
\end{array}\right.\right. \\
& \left\{\begin{array}{l}
\mathbf{T}_{e}\left(\hat{\mathbf{W}}_{p}, \hat{\mathbf{Z}}_{I T}, \hat{\mathbf{Z}}_{P S}, \hat{\delta}_{e}, \hat{\eta}_{e}\right) \succeq \mathbf{0}, \\
\hat{\delta}_{e} \geq 0, \hat{\eta}_{e} \geq 0
\end{array}\right.
\end{aligned}
$$

scheme can asymptotically achieve global optimality at the higher multicast rate region. This is due to the fact that the power allocation for the ANs (i.e., $\mathbf{Z}_{I T}$ and $\mathbf{Z}_{P S}$ ) gradually diminishes with the increase of the multicast rate. Note that problem (29) provides a lower bound on the globally optimal solution to (35) [3].

\section{Rank Revelation Analysis}

In the previous subsection, we proposed globally optimal and sub-optimal solutions to the worst-case SMRRM problem
(20). Now, a natural question arises whether the rank characteristics shown in Section III-B apply to the imperfect CSI case. This question is answered by the following theorem:

Theorem 5: Provided that the optimization problem (28) is feasible for any given $\alpha$, the optimal solution of the confidential service (i.e., $\mathbf{W}_{p}$ ) satisfies $\operatorname{rank}\left(\mathbf{W}_{p}\right) \leq 1$ when $\mathbf{I}-\xi \mathbf{h}_{S I} \mathbf{S I}^{H} \succeq \mathbf{0}$ holds. Moreover, provided that the energy beamforming matrix condition $\mathbf{V} \neq \mathbf{0}$ holds, we always have $\operatorname{rank}(\mathbf{V}) \leq 1$. 
Proof: Please refer to Appendix D.

Remark 3: Suppose that the optimal solution to problem (37), denoted as $\left(\mathbf{V}^{*}, \mathbf{Z}_{P S}^{*}, \mathbf{W}_{0}^{*}, \mathbf{W}_{p}^{*}, \mathbf{Z}_{I T}\right)$ with $\operatorname{rank}\left(\mathbf{W}_{p}^{*}\right)>$ 1 , it is not always possible to construct a solution with the optimal confidential transmission strategy similar to Theorem 3 . Fortunately, we are able to follow the work in [47, Theorem 1] to prove that problem (37) yields the optimal confidential transmission strategy by exploiting its rank-one profile. However, we omit the details of this proof due to space limitation.

\section{NumericAl RESUlts}

In this section, we present simulation results to demonstrate the performance of our proposed schemes. We consider a secure wireless powered FD integrated information service system where the PS is equipped with six transmit antennas $\left(N_{P S}=6\right)$, the FD IT has one receive antenna and six transmit antennas $\left(N_{I T}=6\right)$, an eavesdropper with three receive antennas $\left(N_{E}=3\right)$, and four single-antenna users $(K=4)$. As in [48], we assume that the channel models include both large-scale, i.e., path loss, and small-scale fading, i.e., multi-path fading. Particularly, the simplified large-scale fading model can be expressed as $D=A_{0}\left(\frac{d}{d_{r}}\right)^{-\alpha}, \forall d \geq d_{r}$, where $A_{0}$ is a constant set to $1, \alpha$ is the path loss exponent set to $2.7, d_{r}$ is a reference distance set to 1 meter, and $d$ denotes the propagation distance from the transmitter to the receiver. ${ }^{6}$ On the other hand, the small-scale fading channel coefficients are assumed to be the Rayleigh fading component which obeys $\mathcal{C N}(0, D \mathbf{I})$. Specifically, the channel vector $\mathbf{h}$ from the PS to the FD IT suffers from Rician fading ${ }^{7}$ while the other channel coefficients follow the Rayleigh distribution [48]. Following [43], the coefficients of the self-loop channel vector $\mathbf{h}_{S I}$ are modelled as i.i.d. Rician random variables with a Rician factor of $5 \mathrm{~dB}$. The maximum available power at the PS (i.e., $P_{P S}$ ) and the residual power at the FD IT (i.e., $P_{I T}$ ) are set to be $30 \mathrm{dBm}$ and $0 \mathrm{dBm}$, respectively. The maximum transmit power per-antenna at the PS and the FD IT are set to be $p_{P S}^{n}=$ $p_{I T}^{m}=30 \mathrm{dBm}, \forall n=\left\{1, \ldots, N_{P S}\right\}, \forall m=\left\{1, \ldots, N_{I T}\right\}$ according to [12]. For simplicity, we assume that the noise powers at all receivers are equal to $\sigma_{k}^{2}=\sigma_{e}^{2}=-50 \mathrm{dBm}$, and the energy transfer efficiency is set to be $\xi=0.8$. To highlight the performance tradeoffs of our proposed scheme, in our simulations, we compare our proposed scheme with the following five baseline schemes

1) Baseline 1: We assume that the FD IT transmits no-AN aided integrated service, which means that only the AN

\footnotetext{
${ }^{6}$ This propagation distance includes the PS to the IT (i.e., 5 meters), the PS to users (i.e., 20 meters), the PS to the eavesdropper (i.e., 10 meters), the IT to the users (i.e., 15 meters) and the IT to the eavesdropper (i.e., 5 meters). Note that the transmit power consumption increases, while the secrecy rate decreases as the distance between PS and IT increases [1]. In this paper, it may not be guaranteed to harvest sufficient energy for the FD IT to serve multicast and secrecy unicast services to the users due to severe channel fading conditions as the distance between PS and FD IT increases. In addition, the energy conversion efficiency has an adverse effect on EH with increasing distance between PS and IT. The distance between the PS and the FD IT has been widely considered in the existing literature [1], [47]-[49].

${ }^{7} \mathbf{h}=\sqrt{\frac{K_{R}}{1+K_{R}}} \hat{\mathbf{h}}+\sqrt{\frac{1}{1+K_{R}}} \tilde{\mathbf{h}}$, where $\hat{\mathbf{h}}$ is the line-of-sight (LOS) component with $\|\hat{\mathbf{h}}\|_{2}^{2}=D, \tilde{\mathbf{h}}$ is the Rayleigh fading component denoted by $\tilde{\mathbf{h}} \sim \mathcal{C N}(0, D \mathbf{I})$, and the Rician factor is set to be 3 . Note that the far-field uniform linear antenna array assumption is used to model the LOS component [50].
}

from the PS is used to protect the confidential service transmission.

2) Baseline 2: The PS provides wireless energy to the FD IT only, thus, the AN from the PS is not generated to interfere with the unsubscribed users and the eavesdropper [31].

3) Baseline 3: We assume no energy recycling.

4) Baseline 4 [3], [37]: We assume time division multiple access (TDMA)-based service integration.

5) Baseline 5: We assume the half-duplex (HD) mode in the IT, where the first half of the time block is for energy harvesting from the PS, and the remaining half time block is used for integrated service transmission.

\section{A. Perfect CSI}

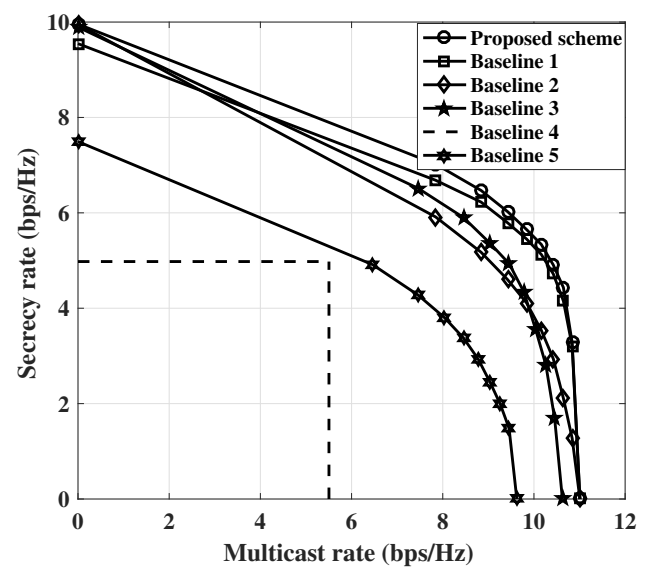

Fig. 2: Secrecy-multicast performance tradeoff with perfect CSI.

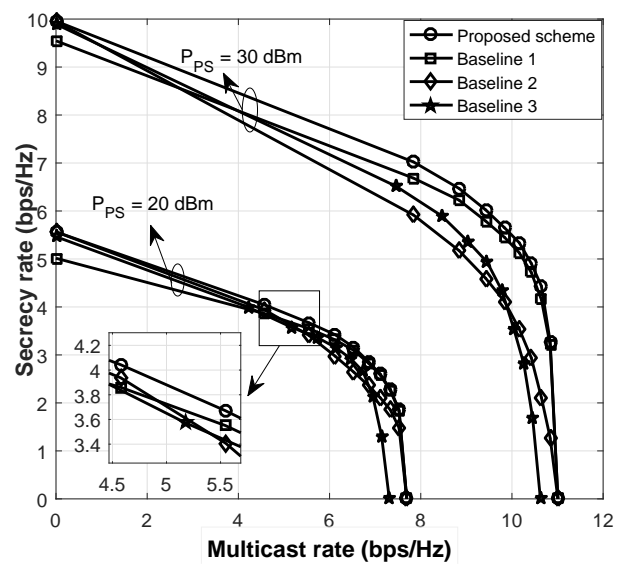

Fig. 3: Secrecy-multicast performance tradeoff with different PS transmit powers.

First, we investigate the performance tradeoff between the secrecy rate (i.e., $\bar{R}_{s e c}$ ) and the multicast rate $\bar{R}_{\text {com }}$ achieved based on the perfect CSI. Fig. 2 shows the secrecy rate versus the multicast rate, which is obtained with $P_{P S}=30 \mathrm{dBm}$. All plots in Fig. 2 are the boundary lines of the secrecymulticast rate regions with different schemes. From this figure, we observe that our proposed scheme outperforms the other five baseline schemes in terms of the secrecy-multicast rate performance tradeoff. In addition, we observe that baseline 1 is closer to the proposed scheme than baseline 2, which 
indicates that the AN from the PS plays a more important role to affect this performance tradeoff than the AN from the FD IT. Moreover, it is also seen that the self-energy recycling at the FD IT can help improve the secrecy-multicast performance tradeoff. As expected, our proposed scheme yields a significantly larger region than the TDMA-based region, which demonstrates the inherent advantage of our proposed scheme over the traditional service integration. Finally, our proposed FD scheme has much better secrecy-multicast performance tradeoff than the HD case, as expected.

Next, we examine the effect of the PS transmit power on the secrecy-multicast performance tradeoff. In this simulation, we compare our proposed scheme with baseline 1, baseline 2, and baseline 3. Fig. 3 shows that the secrecy-multicast performance tradeoff with different PS transmit powers. The same trends and arguments for the secrecy-multicast performance tradeoff from Fig. 2 can be also observed here. Furthermore, one can observe from Fig. 3 that our proposed scheme outperforms baseline 1, baseline 2 and baseline 3 with a lower PS transmit power. In addition, the gap between our proposed scheme and baseline 1 (or baseline 2) becomes smaller when $P_{P S}$ decreases. This is due to the fact that the ANs (i.e., $\mathbf{Z}_{P S}$ and $\mathbf{Z}_{I T}$ ) have to decrease to introduce less interference to all users to guarantee the quality of the multicast service.

\section{B. Imperfect CSI}

Now, we present the results for the imperfect CSI case, where we plot the performance of our proposed schemes together with the suboptimal scheme as well as all baseline schemes. We set $\varepsilon_{1, k}=\varepsilon_{2, k}=\varepsilon_{e, 1}=\varepsilon_{e, 2}=\epsilon$ without loss of generality. Fig. 4 evaluates the worst-case secrecy-multicast performance tradeoff with different channel uncertainties. From this figure, it is observed that the larger the channel uncertainties are, the more degraded worst-case secrecy-multicast performance tradeoff we will get. In addition, we compare our proposed scheme with the suboptimal scheme (c.f. (29)), where the latter provides a lower bound to the former. We also observe that the suboptimal scheme achieves a good approximation to the global secrecy-multicast performance tradeoff. Other observations from Fig. 4 are similar to those drawn from Fig. 2. Specifically, the AN from the PS plays a more significant role in improving the security-multicast rate performance than that of the AN from FD IT. The selfenergy recycling significantly improves the secrecy-multicast performance tradeoff. The comparison between the proposed FD scheme and the associated HD scheme is provided in Fig. 5, where one can observe that the proposed FD selfenergy recycling outperforms the HD scheme in terms of the secrecy-multicast performance tradeoff. Fig. 6 shows that the worst-case secrecy-multicast performance tradeoff with different PS transmit powers. From this figure, the same trends and arguments for the secrecy-multicast performance tradeoff from Fig. 4 can be also observed here. Moreover, with a smaller $P_{P S}$, our proposed scheme exhibits a slightly better performance than baseline 1 and baseline 2, since both $\mathrm{ANs}$ (i.e., $\mathbf{Z}_{P S}$ and $\mathbf{Z}_{I T}$ ) must decrease to reduce the interference introduced to the users such that the quality of the multicast service can be guaranteed. Fig. 7 shows the worstcase secrecy-multicast performance tradeoff versus antenna

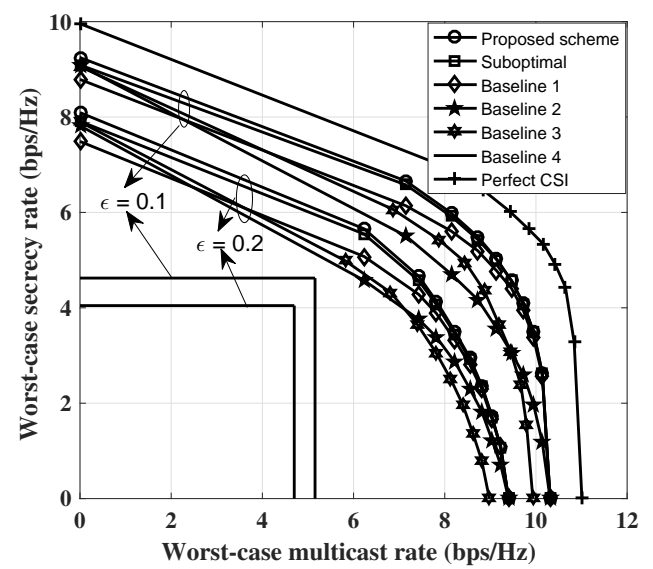

Fig. 4: Secrecy-multicast performance tradeoff with imperfect CSI.

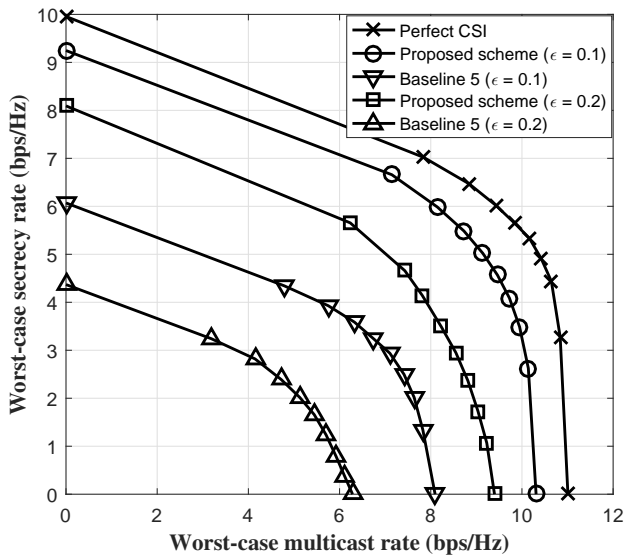

Fig. 5: Worst-case secrecy-multicast performance tradeoff.

configurations with the perfect and imperfect CSI cases. From this figure, it is observed that a larger number of transmit antennas at the FD IT can bring a better secrecy-multicast performance tradeoff, the same conclusion holds as the number of the transmit antennas increases at the PS. This is due to the fact that the receive antenna of the FD IT harvests energy sent from the PS and self-energy recycling from signal leakage of the transmit antenna at the FD IT.

\section{CONCLUSION}

We proposed a novel secure wireless powered integrated service scheme with FD self-energy recycling. The FD IT collects the harvested energy from the PS and its FD selfinterference, respectively, to support the secure integrated services, namely, the multicast and confidential unicast services. We jointly designed the integrated service matrices and the ANs from the PS and FD IT to maximize the achievable secrecy rate region pair. Due to its nonconvexity, we developed an SDP-based two-level approach to solve this optimization problem and found the Pareto optimal points. In addition, we extended our SMRRM problem formulation to the imperfect CSI case, where the worst-case counterpart can be efficiently reduced to a sequence of SDPs by employing quadratic matrix reformulations. Moreover, we analyzed the rank characteristics of the transmit beamforming matrices of the confidential 


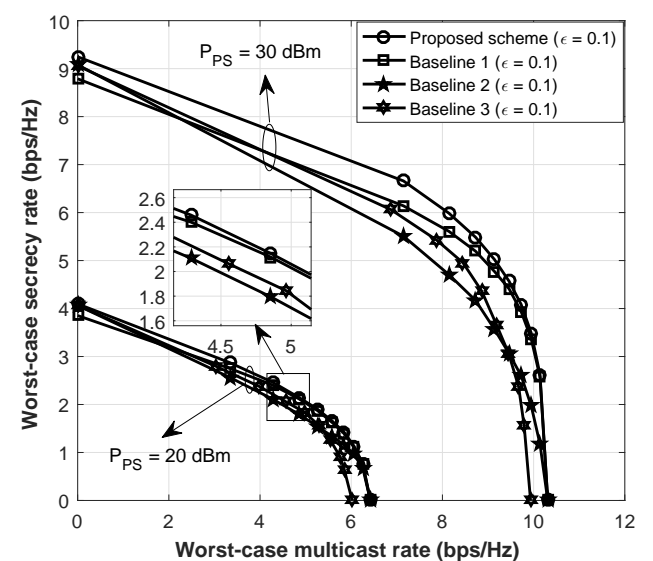

Fig. 6: Worst-case secrecy-multicast performance tradeoff with different PS transmit powers.

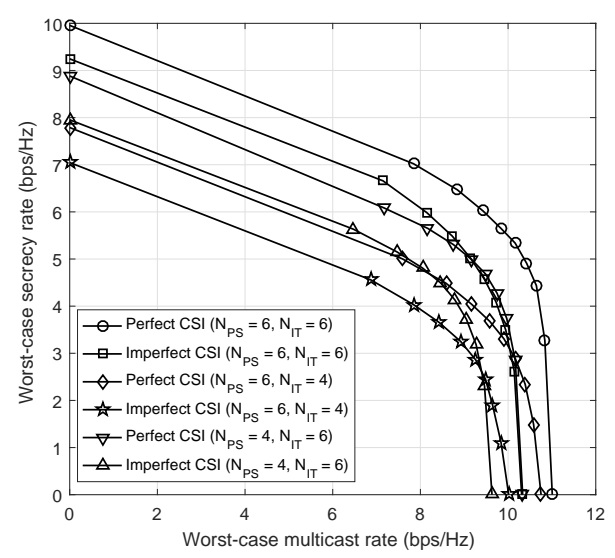

Fig. 7: Secrecy-multicast performance tradeoff with different antenna configurations.

service and the energy transfer for the perfect and imperfect CSI cases. Simulation results were presented to demonstrate the effectiveness of our proposed scheme showing that the ANs and self-energy recycling can improve the secrecy-multicast performance tradeoff. Our proposed scheme also outperforms the traditional TDMA scheme and the HD scheme. Finally, the suboptimal scheme can yield near-optimal performance with a lower complexity. For future works, we will consider a more challenging scenario where the FD IT employs a nonlinear energy harvesting model for wireless charging and self-energy recycling. However, the proposed scheme in this paper cannot be straightforwardly extended to this case. This would change the dynamic of the optimization problems and may require a different approach.

\section{A. Proof of Theorem 2 APPENDIX}

According to (17), we consider the following KKT conditions,

$$
\begin{aligned}
& \mathbf{Y}-\lambda_{1} \mathbf{h}_{1} \mathbf{h}_{1}^{H}=\mathbf{D}, \\
& \mathbf{D} \mathbf{W}_{p}=\mathbf{0}, \\
& \mathbf{D} \succeq \mathbf{0},
\end{aligned}
$$

where $\mathbf{Y}=\mathbf{I}+\sum_{k=2}^{K} \lambda_{k} \mathbf{h}_{k} \mathbf{h}_{k}^{H}+\mathbf{H}_{e} \mathbf{X} \mathbf{H}_{e}^{H}+$ $\sum_{k=1}^{K} \mu_{k} \bar{R}_{c o m}^{\prime} \mathbf{h}_{k} \mathbf{h}_{k}^{H}+\delta\left(\mathbf{I}-\xi \mathbf{h}_{S I} \mathbf{h}_{S I}^{H}\right)+\sum_{m=1}^{N_{I T}} \varphi_{m} \boldsymbol{\Lambda}_{m}$. It is easily observed that the rank of the matrix $\mathbf{Y}$ is a positivedefinite matrix which has full rank when $\mathbf{I}-\xi \mathbf{h}_{S I} \mathbf{h}_{S I}^{H} \succeq \mathbf{0}$ must be satisfied. Thus, when $\mathbf{I}-\xi \mathbf{h}_{S I} \mathbf{h}_{S I}^{H} \succeq \mathbf{0}$ holds, $\operatorname{rank}(\mathbf{Y})=N_{T}$ is satisfied such that we have the following rank relations:

$$
\begin{aligned}
& \operatorname{rank}\left(\mathbf{Y}-\lambda_{1} \mathbf{h}_{1} \mathbf{h}_{1}^{H}\right) \geq \operatorname{rank}(\mathbf{Y})-\operatorname{rank}\left(\lambda_{1} \mathbf{h}_{1} \mathbf{h}_{1}^{H}\right) \\
& \quad \Rightarrow \operatorname{rank}(\mathbf{D}) \geq N_{T}-1 .
\end{aligned}
$$

From (31), we can claim that the rank of $\mathbf{D}$ is either $N_{T}$ or $N_{T}-1$. If $\operatorname{rank}(\mathbf{D})=N_{T}, \mathbf{W}_{p}=\mathbf{0}$ from (30b). However, if $\mathbf{W}_{p}=\mathbf{0}$, it violates our assumption. Thus, $\operatorname{rank}(\mathbf{D})=N_{T}-1$, which satisfies (30b) only when $\mathbf{W}_{p}$ lies in the null space of D whose dimension is one. The first claim in Theorem 2 has been proved.

Now let us focus on the second claim to show that the energy beamforming matrix $\mathbf{V}$ is also rank-one. First, we consider the following KKT conditions:

$$
\begin{aligned}
& \gamma \mathbf{I}+\sum_{n=1}^{N_{P S}} \varrho_{n} \boldsymbol{\Xi}_{n}-\mathbf{M}=\mathbf{A}, \\
& \mathbf{A V}=\mathbf{0}, \\
& \mathbf{A} \succeq \mathbf{0}, \delta \geq 0, \gamma \geq 0,
\end{aligned}
$$

where $\mathbf{M}=\delta \xi \mathbf{h} \mathbf{h}^{H} \succeq \mathbf{0}$, which is a Hermitian matrix. From (32c), the matrix $\mathbf{A}=\gamma \mathbf{I}+\sum_{n=1}^{N_{P S}} \varrho_{n} \boldsymbol{\Xi}_{n}-\mathbf{M} \succeq \mathbf{0}$, $\psi_{\max } \geq \kappa \geq 0$ holds, where $\psi_{\max }$ and $\kappa$ are the real-valued maximum eigenvalues of the matrices $\gamma \mathbf{I}+\sum_{n=1}^{N_{P S}} \varrho_{n} \boldsymbol{\Xi}_{n}$ and $\mathbf{M}$, respectively. Considering the KKT condition (32b), we can show that if $\psi_{\max }>\kappa$, the matrix $\mathbf{A}$ is positive definite and full-rank. However, this will lead to the optimal solution $\mathbf{V}=\mathbf{0}$ which contradicts our assumption. Thus, to satisfy the complementary slackness condition (32b), we can claim that either $\mathbf{V}^{\text {opt }}=\mathbf{0}$ or $\operatorname{rank}\left(\mathbf{V}^{\text {opt }}\right) \leq 1$ holds at the optimal solution. $^{8}$

Thus, the dual variable $\psi_{\max }$ has to be equal to the eigenvalue of $\mathbf{M}$, i.e., $\psi_{\max }=\kappa$. In order to have a bounded optimal dual solution, it follows that the null space of $\mathbf{A}$ is spanned by a vector $\mathbf{p}$, which is the unit-norm eigenvector of $\mathbf{M}$ associated with the eigenvalue $\kappa$. As a result, we can claim that $\operatorname{rank}(\mathbf{A}) \geq N_{P S}-1$. Thus, $\operatorname{rank}(\mathbf{V}) \leq 1$ holds.

\section{B. Proof of Theorem 3}

First, it is verified that problem (15) is jointly convex in terms of the primal variables (i.e., $\mathbf{V}, \mathbf{Z}_{P S}, \mathbf{W}_{0}, \mathbf{W}_{p}, \mathbf{Z}_{I T}$ ) and satisfies the Slater's condition [41]. Thus, the gap between the primal and dual optimization problems is zero. According to (17), the dual optimization problem is given by

$$
\max _{\substack{\lambda_{k}, \mu_{k}, \delta, \gamma, \varrho_{n}, \varphi_{m} \geq 0 \\ \mathbf{A}, \mathbf{B}, \mathbf{C}, \mathbf{D}, \mathbf{F}, \mathbf{X} \succeq \mathbf{0}}} \min _{\mathbf{W}_{0}, \mathbf{Z}_{p}, \mathbf{Z}_{I T} \succeq \mathbf{0}} \mathcal{L}(\boldsymbol{\Omega}),
$$

which achieves the same objective value with its relative primal optimization problem (15). By exploiting the dual problem (33), we obtain the primal and dual optimal solution of (15) and (33), denoted as, $\left(\mathbf{V}^{*}, \mathbf{Z}_{P S}^{*}, \mathbf{W}_{0}^{*}, \mathbf{W}_{p}^{*}, \mathbf{Z}_{I T}^{*}\right)$

${ }^{8}$ When $\mathbf{V}^{\text {opt }}=\mathbf{0}$, it follows that the IT has sufficient stored energy and does not need to be wirelessly charged, the FD energy recycle and the AN (i.e., $\mathbf{Z}_{P S}$ ) from the PS are employed to support secure integrated service transmission. 
and $\left(\lambda_{k}^{*}, \mu_{k}^{*}, \delta^{*}, \gamma^{*}, \varrho_{n}^{*}, \varphi_{m}^{*}, \mathbf{A}^{*}, \mathbf{B}^{*}, \mathbf{C}^{*}, \mathbf{D}^{*}, \mathbf{F}^{*}, \mathbf{X}^{*}\right)$, respectively. From (30a), we can claim that the rank of matrix $\mathbf{Y}^{*}$ is indeterminate. Hence, two scenarios are discussed in the following. Let us denote $\operatorname{rank}(\mathbf{Y})=r$. First, we consider the case that $r=N_{T}$, which implies that $\mathbf{Y}$ is a full-rank matrix, such that we can easily show that the optimal solution $\mathbf{W}_{p}$ is rank-one (c.f. Proof of Theorem 2). Additionally, the optimal solution of the confidential service matrix $\mathbf{W}_{p}^{*}$ is expressed as $\mathbf{W}_{p}^{*}=c_{0} \mathbf{q}_{0} \mathbf{q}_{0}^{H}$, where $c_{0}>0$ is a constant and $\mathbf{q}_{0} \in \mathbb{C}^{N_{I T} \times 1}$ spans the null space of $\mathbf{D}^{*}$.

Next, we investigate the case $r<N_{T}$, which implies that the matrix $\mathbf{Y}$ is not full-rank. In this case, we exploit the structure of $\mathbf{W}_{p}^{*}$ by constructing a new solution for problem (15) with $\operatorname{rank}\left(\mathbf{W}_{p}^{*}\right)=1$. It is assumed that $\Theta \in \mathbb{C}^{N_{T} \times N_{T}-r}$ with $\boldsymbol{\Theta}^{H} \boldsymbol{\Theta}=\mathbf{I}$ contains the orthonormal basis vector of the null space of $\mathbf{Y}$, i.e., $\mathbf{Y} \boldsymbol{\Theta}=\mathbf{0}$, and $\operatorname{rank}(\boldsymbol{\Theta})=N_{T}-r$.

Denote by $\mathbf{q}_{n} \in \mathbb{C}^{N_{T} \times 1}, 1 \leq n \leq N_{T}-r$ the $n$-th column of $\boldsymbol{\Theta}$. Then, we have $\mathbf{q}_{n}^{H} \mathbf{D} \mathbf{q}_{n}=\mathbf{q}_{n}^{H}\left(\mathbf{Y}-\lambda_{1} \mathbf{h}_{1} \mathbf{h}_{1}^{H}\right) \mathbf{q}_{n}=$ $-\lambda_{1}\left|\mathbf{h}_{1}^{H} \mathbf{q}_{n}\right|^{2}, 1 \leq n \leq N_{T}-r$. Due to the constraint (16) and the KKT condition (30c), we have $\lambda_{1}>0$ and $\mathbf{D} \succeq \mathbf{0}$ such that $\mathbf{h}_{1} \mathbf{h}_{1}^{H} \boldsymbol{\Theta}=\mathbf{0}$. Thus, we have $\mathbf{D} \boldsymbol{\Theta}=\left(\mathbf{Y}-\lambda_{1} \mathbf{h}_{1} \mathbf{h}_{1}^{H}\right) \boldsymbol{\Theta}=\mathbf{0}$. Additionally, from (30a), we have $\operatorname{rank}(\mathbf{D}) \geq \operatorname{rank}(\mathbf{Y})-$ $\operatorname{rank}\left(\lambda_{1} \mathbf{h}_{1} \mathbf{h}_{1}^{H}\right)=r-1$. Moreover, $\operatorname{since} \operatorname{rank}(\overline{\boldsymbol{\Theta}})=N_{T}-r$, it follows that $\operatorname{rank}(\mathbf{D}) \leq r$. Thus, we can conclude that $N_{T}-r \leq \operatorname{rank}\left(\mathbf{W}_{p}^{*}\right) \leq N_{T}-r+1$ due to (30b). Now, suppose that $\operatorname{rank}\left(\mathbf{W}_{p}\right)=\operatorname{rank}(\boldsymbol{\Theta})=N_{T}-r$, then we have $\mathbf{W}_{p}=\boldsymbol{\Theta}$. From (30b) and (30c), the optimal solution of the confidential service matrix $\mathbf{W}_{p}^{*}$ can be written as $\mathbf{W}_{p}^{*}=\sum_{n=1}^{N_{T}-r} d_{n} \mathbf{q}_{n} \mathbf{q}_{n}^{H}$, where $d_{n}>0, \forall n$. However, this case implies that no confidential service has been ordered by user 1 due to the fact that each $\mathbf{q}_{n}$ lies in the null space of $\mathbf{h}_{1}$. Hence, it follows that $\operatorname{rank}\left(\mathbf{W}_{p}\right)=N_{T}-r+1$, and there exists only one single subspace spanned by $\mathbf{q}_{0} \in \mathbb{C}^{N_{T} \times 1}$ of unit norm, which lies in the null space of $\mathbf{D}$, i.e., $\mathbf{D} \mathbf{q}_{0}=\mathbf{0}$, and is orthogonal to the span of $\boldsymbol{\Theta}$, i.e., $\boldsymbol{\Theta}^{H} \mathbf{q}_{0}=\mathbf{0}$. Thus, we obtain the structure of the confidential service optimal solution as $\mathbf{W}_{p}^{*}=\left[\boldsymbol{\Theta} \mathbf{q}_{0}\right]$, and $\operatorname{rank}\left(\mathbf{W}_{p}^{*}\right)=N_{T}-r+1$. We can express the optimal solution of problem (15) as $\mathbf{W}_{p}^{*}=\sum_{n=1}^{N_{T}-r} d_{n} \mathbf{q}_{n} \mathbf{q}_{n}^{H}+c_{0} \mathbf{q}_{0} \mathbf{q}_{0}^{H}$. Suppose that $\left(\mathbf{V}^{*}, \mathbf{Z}_{P S}^{*}, \mathbf{W}_{0}^{*}, \mathbf{W}_{p}^{*}, \mathbf{Z}_{I T}^{*}\right)$ is the optimal solution to problem (15) with $\operatorname{rank}\left(\mathbf{W}_{P}^{\text {opt }}\right) \geq 1$, we can reconstruct a new solution $\left(\tilde{\mathbf{V}}^{*}, \tilde{\mathbf{Z}}_{P S}^{*}, \tilde{\mathbf{W}}_{0}^{*}, \mathbf{W}_{p}^{*}, \tilde{\mathbf{Z}}_{I T}^{*}\right)$ given by

$$
\begin{aligned}
\tilde{\mathbf{W}}_{p}^{*} & =\mathbf{W}_{p}^{*}-\sum_{n=1}^{N_{T}-r} d_{n} \mathbf{q}_{n} \mathbf{q}_{n}^{H}=c_{0} \mathbf{q}_{0} \mathbf{q}_{0}, \\
\tilde{\mathbf{Z}}_{I T}^{*} & =\mathbf{Z}_{I T}^{*}+\sum_{n=1}^{N_{T}-r} d_{n} \mathbf{q}_{n} \mathbf{q}_{n}^{H}, \quad \tilde{\mathbf{W}}_{0}^{*}=\mathbf{W}_{0}^{*}, \\
\tilde{\mathbf{V}}^{*} & =\mathbf{V}^{*}, \tilde{\mathbf{Z}}_{P S}^{*}=\mathbf{Z}_{P S}^{*} .
\end{aligned}
$$

We substitute the new optimal solutions in (34) into the objective function and all constraints in (15), it can be observed that the reconstructed solution is also feasible for problem (15) and achieves the same optimal value as $\left(\mathbf{V}^{*}, \mathbf{Z}_{P S}^{*}, \mathbf{W}_{0}^{*}, \mathbf{W}_{p}^{*}, \mathbf{Z}_{I T}^{*}\right)$ such that $\operatorname{rank}\left(\overline{\mathbf{W}}_{p}\right)=1$ (c.f. (34a)).

\section{Proof of Theorem 4}

First, we define

$$
\begin{aligned}
& f_{1}\left(\mathbf{W}_{p}, \mathbf{Z}_{I T}, \mathbf{Z}_{P S}\right) \\
& =\min _{\substack{\mathbf{h}_{1} \in \mathcal{S}_{1,1} \\
\mathbf{g}_{1} \in \mathcal{S}_{2,1}}} \frac{\mathbf{h}_{1}^{H}\left(\mathbf{W}_{p}+\mathbf{Z}_{I T}\right) \mathbf{h}_{1}+\mathbf{g}_{1}^{H} \mathbf{Z}_{P S} \mathbf{g}_{1}+\sigma_{1}^{2}}{\alpha_{2}\left(\mathbf{h}_{1}^{H} \mathbf{Z}_{I T} \mathbf{h}_{1}+\mathbf{g}_{1}^{H} \mathbf{Z}_{P S} \mathbf{g}_{1}+\sigma_{1}^{2}\right)} .
\end{aligned}
$$

If we show that (28) is a quasi-convex, then we need to show that $f_{1}$ is a quasi-concave function on the problem domain in (28), which will be our focus in the remaining part of this proof. With the convexity of the problem domain in (28) established, we should check whether all the $\tilde{\alpha}$-superlevel sets of $f_{1}$ are convex for every $\tilde{\alpha}$ [41]. The $\tilde{\alpha}$-superlevel set of $f_{1}$ is defined as $\mathcal{S}_{\tilde{\alpha}}=\left\{\left(\mathbf{W}_{p}, \mathbf{Z}_{I T}, \mathbf{Z}_{P S}\right) \mid \mathbf{W}_{p} \succeq \mathbf{0}, \mathbf{Z}_{I T} \succeq\right.$ $\left.\mathbf{0}, \mathbf{Z}_{P S} \succeq \mathbf{0}, f_{1} \geq \tilde{\alpha}\right\}$. Next, we reveal the convexity of $f_{1} \geq \tilde{\alpha}$ by resorting to the S-Procedure and [46, Theorem 3.5], (28a) is reformulated as the following LMI:

$$
\left[\begin{array}{ccc}
\bar{\beta}_{1} \mathbf{I}_{N_{I T}}+\mathbf{D}_{1} & \mathbf{D}_{1} \overline{\mathbf{h}}_{1} & \mathbf{0}_{N_{I T} \times N_{P S}} \\
\overline{\mathbf{h}}_{1}^{H} \mathbf{D}_{1} & t_{1} & \overline{\mathbf{g}}_{1}^{H} \mathbf{D}_{2} \\
\mathbf{0}_{N_{P S} \times N_{I T}} & \mathbf{D}_{2} \overline{\mathbf{g}}_{1} & \mathbf{D}_{2}+\frac{\bar{\theta}_{1}}{\varepsilon_{2,1}^{2}} \mathbf{I}_{N_{P S}}
\end{array}\right] \succeq \mathbf{0},
$$

where $\bar{\beta}_{1} \geq 0, \bar{\theta}_{1} \geq 0, \mathbf{D}_{1}=\mathbf{W}_{p}+\left(1-\tilde{\alpha} \alpha_{2}\right) \mathbf{Z}_{I T}$, $\mathbf{D}_{2}=\left(1-\tilde{\alpha} \alpha_{2}\right) \mathbf{Z}_{P S}$, and $t_{1}=\overline{\mathbf{h}}_{1}^{H} \mathbf{D}_{1} \overline{\mathbf{h}}_{1}+\overline{\mathbf{g}}_{1}^{H} \mathbf{D}_{2} \overline{\mathbf{g}}_{1}+(1-$ $\left.\tilde{\alpha} \alpha_{2}\right) \sigma_{1}^{2}-\bar{\beta}_{1} \varepsilon_{1,1}^{2}-\bar{\theta}_{1}$. The LMI (36) is convex in terms of $\left(\mathbf{W}_{p}, \mathbf{Z}_{I T}, \mathbf{Z}_{P S}, \bar{\beta}_{1}, \bar{\theta}_{1}\right)$. Hence, $\mathcal{S}_{\tilde{\alpha}}$ is a convex set for every $\tilde{\alpha}$, and we conclude that $f_{1}$ is a quasi-concave function.

\section{Proof of Theorem 5}

Let $g_{w c}^{\mathrm{opt}}\left(\bar{R}_{c o m, w c}, \alpha_{2}\right)$ be the optimal objective function value of (28). Similar to Section III-B, we consider the following power minimization problem (37) on the top of the next page, where $\bar{s}_{0, k}=-\bar{R}_{c o m, w c}^{\prime} \sigma_{k}^{2}-\bar{\lambda}_{k} \varepsilon_{1, k}^{2}-\bar{\gamma}_{k}, \bar{t}_{0, k}=$ $\left(\alpha_{2}-1\right) \sigma_{k}^{2}-\bar{\beta}_{k} \varepsilon_{1, k}^{2}-\bar{\theta}_{k}, \bar{t}_{1}=\left[1-g_{w c}^{\mathrm{opt}}\left(\bar{R}_{c o m, w c}^{\prime}, \alpha_{2}\right) \alpha_{2}\right]$ $\sigma_{1}^{2}-\bar{\beta}_{1} \varepsilon_{1,1}^{2}-\bar{\theta}_{1}, \mathbf{R}_{1}=\mathbf{W}_{p}+\left(1-g_{w c}^{\mathrm{opt}}\left(\bar{R}_{c o m, w c}^{\prime}, \alpha_{2}\right) \alpha_{2}\right) \mathbf{Z}_{I T}$, $\mathbf{R}_{2}=\left(1-g_{w c}^{\mathrm{opt}}\left(\bar{R}_{c o m, w c}^{\prime}, \alpha_{2}\right) \alpha_{2}\right) \mathbf{Z}_{P S}$, also,

$$
\begin{aligned}
\mathbf{S}_{0, k}= & {\left[\begin{array}{ccc}
\bar{\lambda}_{k} \mathbf{I}_{N_{I T}} & \mathbf{0}_{N_{I T} \times 1} & \mathbf{0}_{N_{I T} \times N_{P S}} \\
\mathbf{0}_{1 \times N_{I T}} & \bar{s}_{0, k} & \mathbf{0}_{1 \times N_{P S}} \\
\mathbf{0}_{N_{P S} \times N_{I T}} & \mathbf{0}_{N_{P S} \times 1} & \frac{\bar{\gamma}_{k}}{\varepsilon_{2, k}^{2}} \mathbf{I}_{N_{P S}}
\end{array}\right], \forall k \in \mathcal{K}, } \\
\mathbf{T}_{0, k}= & {\left[\begin{array}{ccc}
\bar{\beta}_{k} \mathbf{I}_{N_{I T}} & \mathbf{0}_{N_{I T} \times 1} & \mathbf{0}_{N_{I T} \times N_{P S}} \\
\mathbf{0}_{1 \times N_{I T}} & \bar{t}_{0, k} & \mathbf{0}_{1 \times N_{P S}} \\
\mathbf{0}_{N_{P S} \times N_{I T}} & \mathbf{0}_{N_{P S} \times 1} & \frac{\bar{\theta}_{k}}{\varepsilon_{2, k}^{2}} \mathbf{I}_{N_{P S}}
\end{array}\right], \forall k \in \overline{\mathcal{K}}, } \\
\mathbf{T}_{0, e}= & {\left[\begin{array}{ccc}
\frac{\bar{\delta}_{e}}{\varepsilon_{1, e}^{2}} \mathbf{I}_{N_{I T}} & \mathbf{0}_{N_{I T} \times N_{E}} & \mathbf{0}_{N_{I T} \times N_{P S}} \\
\mathbf{0}_{N_{E} \times N_{I T}} & {\left[\left(\alpha_{2}-1\right) \sigma_{e}^{2}-\bar{\delta}_{e}-\bar{\eta}_{e}\right] \mathbf{I}_{N_{E}}} & \mathbf{0}_{N_{E} \times N_{P S}} \\
\mathbf{0}_{N_{P S} \times N_{I T}} & \mathbf{0}_{N_{P S} \times N_{E}} & \frac{\bar{\eta}_{e}}{\varepsilon_{2, e}} \mathbf{I}_{N_{P S}}
\end{array}\right], } \\
\mathbf{T}_{0,1}= & {\left[\begin{array}{ccc}
\bar{\beta}_{1} \mathbf{I}_{N_{I T}} & \mathbf{0}_{N_{I T} \times 1} & \mathbf{0}_{N_{I T} \times N_{P S}} \\
\mathbf{0}_{1 \times N_{I T}} & \bar{t}_{1} & \mathbf{0}_{1 \times N_{P S}} \\
\mathbf{0}_{N_{P S} \times N_{I T}} & \mathbf{0}_{N_{P S} \times 1} & \frac{\bar{\theta}_{1}}{\varepsilon_{2,1}^{2}} \mathbf{I}_{N_{P S}}
\end{array}\right], } \\
\hat{\mathbf{H}}_{k}= & {\left[\begin{array}{lll}
\mathbf{I}_{N_{I T}} & \overline{\mathbf{h}}_{k} & \mathbf{0}_{N_{I T} \times N_{P S}}
\end{array}\right], } \\
\hat{\mathbf{G}}_{k}= & {\left[\begin{array}{lll}
\mathbf{0}_{N_{P S} \times N_{I T}} & \overline{\mathbf{g}}_{k} \mathbf{I}_{N_{P S}}
\end{array}\right], \forall k \in \mathcal{K} . }
\end{aligned}
$$

We can claim that (37) shares the same optimal solution with (28). Consequently, we investigate the Lagrange dual function of (37), which is written 


$$
\begin{aligned}
& \min _{\mathbf{V}_{, \mathbf{Z}_{P S}}, \mathbf{W}_{0}, \mathbf{W}_{p}, \mathbf{Z}_{I T}} \operatorname{Tr}\left(\mathbf{W}_{0}+\mathbf{W}_{p}+\mathbf{Z}_{I T}\right), \text { s.t.(5a), (5b), (6a), (6b), }(5 \mathrm{e}),\left\{\bar{\lambda}_{k}, \bar{\gamma}_{k}, \bar{\beta}_{k}, \bar{\theta}_{k}, \bar{\delta}_{e}, \bar{\eta}_{e}\right\} \geq 0, \forall k \in \mathcal{K}, \\
& \mathbf{S}_{0, k}+\hat{\mathbf{H}}_{k}^{H}\left[\mathbf{W}_{0}-\bar{R}_{c o m, w c}^{\prime}\left(\mathbf{W}_{p}+\mathbf{Z}_{I T}\right)\right] \hat{\mathbf{H}}_{k}-\hat{\mathbf{G}}_{k}^{H}\left(\hat{R}_{c o m, w c}^{\prime} \mathbf{Z}_{P S}\right) \hat{\mathbf{G}}_{k} \succeq \mathbf{0}, \forall k \in \mathcal{K}, \\
& \mathbf{T}_{0, k}-\hat{\mathbf{H}}_{k}^{H} \overline{\mathbf{B}}_{1} \hat{\mathbf{H}}_{k}+\hat{\mathbf{G}}_{k}^{H} \overline{\mathbf{B}}_{2} \hat{\mathbf{G}}_{k} \succeq \mathbf{0}, \forall k \in \overline{\mathcal{K}}, \mathbf{T}_{0, e}+\hat{\mathbf{H}}_{e}^{H} \overline{\mathbf{C}}_{1} \hat{\mathbf{H}}_{e}+\hat{\mathbf{G}}_{e}^{H} \overline{\mathbf{C}}_{2} \hat{\mathbf{G}}_{e} \succeq \mathbf{0}, \\
& \mathbf{T}_{0,1}+\hat{\mathbf{H}}_{1}^{H} \mathbf{R}_{1} \hat{\mathbf{H}}_{1}+\hat{\mathbf{G}}_{1}^{H} \mathbf{R}_{2} \hat{\mathbf{G}}_{1} \succeq \mathbf{0},
\end{aligned}
$$

$$
\begin{aligned}
& \mathcal{L}\left(\boldsymbol{\Omega}_{2}\right)=\operatorname{Tr}\left(\mathbf{W}_{0}\right)+\operatorname{Tr}\left(\mathbf{W}_{p}\right)+\operatorname{Tr}\left(\mathbf{Z}_{I T}\right)-\operatorname{Tr}(\mathbf{A V})-\operatorname{Tr}\left(\mathbf{B} \mathbf{Z}_{P S}\right)-\operatorname{Tr}\left(\mathbf{C W}_{0}\right)-\operatorname{Tr}\left(\mathbf{D} \mathbf{W}_{p}\right)-\operatorname{Tr}\left(\mathbf{F} \mathbf{Z}_{I T}\right) \\
& +\delta\left[\operatorname{Tr}\left[\left(\mathbf{I}-\xi \mathbf{h}_{S I} \mathbf{h}_{S I}^{H}\right)\left(\mathbf{W}_{0}+\mathbf{W}_{p}+\mathbf{Z}_{I T}\right)\right]-P_{I T}-\xi \operatorname{Tr}\left[\mathbf{h} \mathbf{h}^{H}\left(\mathbf{V}+\mathbf{Z}_{P S}\right)\right]\right]+\gamma\left[\operatorname{Tr}\left(\mathbf{V}+\mathbf{Z}_{P S}\right)-P_{P S}\right] \\
& +\sum_{n=1}^{N_{P S}} \varrho_{n}\left[\operatorname{Tr}\left[\mathbf{\Xi}_{n}\left(\mathbf{V}+\mathbf{Z}_{P S}\right)\right]-p_{P S}^{n}\right]+\sum_{m=1}^{N_{I T}} \varphi_{m}\left[\operatorname{Tr}\left[\mathbf{\Lambda}_{m}\left(\mathbf{W}_{0}+\mathbf{W}_{p}+\mathbf{Z}_{I T}\right)\right]-p_{I T}^{m}\right]-\sum_{k=1}^{K} \operatorname{Tr}\left[\mathbf { X } _ { k } \left(\mathbf{S}_{0, k}\right.\right. \\
& \left.\left.+\hat{\mathbf{H}}_{k}^{H} \overline{\mathbf{A}}_{1} \hat{\mathbf{H}}_{k}-\hat{\mathbf{G}}_{k}^{H} \overline{\mathbf{A}}_{2} \hat{\mathbf{G}}_{k}\right)\right]-\sum_{k=2}^{K} \operatorname{Tr}\left[\mathbf{Y}_{k}\left(\mathbf{T}_{0, k}-\hat{\mathbf{H}}_{k}^{H} \overline{\mathbf{B}}_{1} \hat{\mathbf{H}}_{k}+\hat{\mathbf{G}}_{k}^{H} \overline{\mathbf{B}}_{2} \hat{\mathbf{G}}_{k}\right)\right]-\operatorname{Tr}\left[\mathbf { Y } _ { e } \left(\mathbf{T}_{e}\right.\right. \\
& \left.\left.+\hat{\mathbf{H}}_{e}^{H} \overline{\mathbf{C}}_{1} \hat{\mathbf{H}}_{e}+\hat{\mathbf{G}}_{e}^{H} \overline{\mathbf{C}}_{2} \hat{\mathbf{G}}_{e}\right)\right]-\operatorname{Tr}\left[\mathbf{Y}_{1}\left(\mathbf{T}_{0,1}+\hat{\mathbf{H}}_{1}^{H} \mathbf{R}_{1} \hat{\mathbf{H}}_{1}+\hat{\mathbf{G}}_{1}^{H} \mathbf{R}_{2} \hat{\mathbf{G}}_{1}\right)\right] .
\end{aligned}
$$

as (38) on the top of the this page, where $\Omega_{2}=$ nonsingular matrix does not affect the matrix rank. The $\left\{\mathbf{V}, \mathbf{Z}_{P S}, \mathbf{W}_{0}, \mathbf{W}_{p}, \mathbf{Z}_{I T}, \mathbf{A}, \mathbf{B}, \mathbf{C}, \mathbf{D}, \mathbf{F}, \delta, \gamma, \varrho_{n}, \varphi_{m}, \mathbf{X}_{k}, \mathbf{Y}_{k}\right.$, Yfolllowing rank relation holds,

$\mathbf{A} \succeq \mathbf{0}, \mathbf{B} \succeq \mathbf{0}, \mathbf{C} \succeq \mathbf{0}, \mathbf{D} \succeq \mathbf{0}, \mathbf{F} \succeq \mathbf{0}, \mathbf{X}_{k} \succeq \mathbf{0}, \mathbf{Y}_{k} \succeq \mathbf{0}$, $\mathbf{Y}_{e} \succeq \mathbf{0}, \delta \geq 0, \gamma \geq 0, \varrho_{n} \geq 0$, and $\varphi_{m} \geq 0$ are dual variables pertaining to the primary constraints in (37). To proceed, we consider the following KKT conditions:

$$
\begin{aligned}
& \mathbf{D}=\mathbf{Y}-\hat{\mathbf{H}}_{1} \mathbf{Y}_{1} \hat{\mathbf{H}}_{1}^{H}, \\
& \mathbf{D} \mathbf{W}_{p}=\mathbf{0}, \\
& \mathbf{Y}_{1}\left(\mathbf{T}_{0,1}+\hat{\mathbf{H}}_{1}^{H} \mathbf{R}_{1} \hat{\mathbf{H}}_{1}+\hat{\mathbf{G}}_{1}^{H} \mathbf{R}_{2} \hat{\mathbf{G}}_{1}\right)=\mathbf{0},
\end{aligned}
$$

where $\mathbf{Y}=\mathbf{I}+\delta\left(\mathbf{I}-\xi \mathbf{h}_{S I} \mathbf{h}_{S I}^{H}\right)+\sum_{m=1}^{N_{I T}} \varphi_{m} \boldsymbol{\Lambda}_{m}+$ $\sum_{k=1}^{K} \hat{\mathbf{H}}_{k} \mathbf{X}_{k} \hat{\mathbf{H}}_{k}^{H}+\sum_{k=2}^{K} \hat{\mathbf{H}}_{k} \mathbf{Y}_{k} \hat{\mathbf{H}}_{k}^{H}+\hat{\mathbf{H}}_{e} \mathbf{Y}_{e} \hat{\mathbf{H}}_{e}^{H}$. Postmultiplying (39a) by $\mathbf{W}_{p}$ and applying (39b) yields $\mathbf{Y} \mathbf{W}_{p}=$ $\hat{\mathbf{H}}_{1} \mathbf{Y}_{1} \hat{\mathbf{H}}_{1}^{H} \mathbf{W}_{p}$. It is shown that $\mathbf{Y} \succ \mathbf{0}$ when $\mathbf{I}-\xi \mathbf{h}_{S I} \mathbf{h}_{S I}^{H} \succeq \mathbf{0}$ holds. Thus, we have the following rank relations

$$
\operatorname{rank}\left(\mathbf{W}_{p}\right)=\operatorname{rank}\left(\hat{\mathbf{H}}_{1} \mathbf{Y}_{1} \hat{\mathbf{H}}_{1}^{H} \mathbf{W}_{p}\right) \leq \operatorname{rank}\left(\hat{\mathbf{H}}_{1} \mathbf{Y}_{1} \hat{\mathbf{H}}_{1}^{H}\right) .
$$

Thus, the remaining part of this proof is to focus on the rank of $\hat{\mathbf{H}}_{1} \mathbf{Y}_{1} \hat{\mathbf{H}}_{1}^{H}$. We premultiply and postmultiply (39c) by $\hat{\mathbf{H}}_{1}$ and $\hat{\mathbf{H}}=\left[\begin{array}{lll}\mathbf{I}_{N_{I T}} & \mathbf{0}_{N_{I T} \times 1} & \mathbf{0}_{N_{I T} \times N_{P S}}\end{array}\right]^{H}$, respectively, to get

$$
\begin{aligned}
\hat{\mathbf{H}}_{1} \mathbf{Y}_{1} \mathbf{T}_{0,1} \hat{\mathbf{H}}+\hat{\mathbf{H}}_{1} \mathbf{Y}_{1} \hat{\mathbf{H}}_{1}^{H} \mathbf{R}_{1} \hat{\mathbf{H}}_{1} \hat{\mathbf{H}} & \\
& +\hat{\mathbf{H}}_{1} \mathbf{Y}_{1} \hat{\mathbf{G}}_{1}^{H} \mathbf{R}_{2} \hat{\mathbf{G}}_{1} \hat{\mathbf{H}}=\mathbf{0}_{N_{I T} \times N_{I T}},
\end{aligned}
$$

where $\mathbf{R}_{1}=\mathbf{W}_{p}+\left(1-g_{w c}^{\mathrm{opt}}\left(\bar{R}_{c o m, w c}^{\prime}, \alpha_{2}\right) \alpha_{2}\right) \mathbf{Z}_{I T}$, and $\mathbf{R}_{2}=\left(1-g_{w c}^{\mathrm{opt}}\left(\bar{R}_{c o m, w c}^{\prime}, \alpha_{2}\right) \alpha_{2}\right) \mathbf{Z}_{P S}$. To exploit the rank of $\hat{\mathbf{H}}_{1} \mathbf{Y}_{1} \hat{\mathbf{H}}_{1}^{H}$, we consider the following equalities

$$
\mathbf{T}_{0,1} \hat{\mathbf{H}}=\bar{\beta}_{1}\left(\hat{\mathbf{H}}_{1}^{H}-\hat{\mathbf{h}}_{1}^{H}\right), \hat{\mathbf{H}}_{1} \hat{\mathbf{H}}=\mathbf{I}_{N_{I T}}, \overline{\mathbf{G}}_{1} \hat{\mathbf{H}}=\mathbf{0}_{N_{P S} \times N_{I T}},
$$

where $\hat{\mathbf{h}}_{1}=\left[\mathbf{0}_{N_{I T}} \overline{\mathbf{h}}_{1} \mathbf{0}_{N_{I T} \times N_{P S}}\right]$. Substituting (42) into (41) yields $\hat{\mathbf{H}}_{1} \mathbf{Y}_{1} \hat{\mathbf{H}}_{1}^{H}\left(\mathbf{R}_{1}+\bar{\beta}_{1} \mathbf{I}_{N_{I T}}\right)=\bar{\beta}_{1} \hat{\mathbf{H}}_{1} \mathbf{Y}_{1} \hat{\mathbf{h}}_{1}^{H}$. It is easily verified that $\mathbf{R}_{1}+\bar{\beta}_{1} \mathbf{I}_{N_{I T}}$ is a positive semidefinite matrix and nonsingular, thus, pre or postmultiplying by a

$$
\begin{array}{r}
\operatorname{rank}\left(\hat{\mathbf{H}}_{1} \mathbf{Y}_{1} \hat{\mathbf{H}}_{1}^{H}\right)=\operatorname{rank}\left(\hat{\mathbf{H}}_{1} \mathbf{Y}_{1} \hat{\mathbf{H}}_{1}^{H}\left(\mathbf{R}_{1}+\bar{\beta}_{1} \mathbf{I}_{N_{I T}}\right)\right) \\
=\operatorname{rank}\left(\bar{\beta}_{1} \hat{\mathbf{H}}_{1} \mathbf{Y}_{1} \hat{\mathbf{h}}_{1}^{H}\right) \leq \operatorname{rank}\left(\hat{\mathbf{h}}_{1}^{H}\right) \leq 1 .
\end{array}
$$

With the relations (40) and (43), we obtain $\operatorname{rank}\left(\mathbf{W}_{p}\right) \leq$ $\operatorname{rank}\left(\hat{\mathbf{H}}_{1} \mathbf{Y}_{1} \hat{\mathbf{H}}_{1}^{H}\right) \leq 1$. We have either $\mathbf{W}_{p}=\mathbf{0}$ or $\operatorname{rank}\left(\mathbf{W}_{p}\right)=1$. Suppose that $\mathbf{W}_{p}=\mathbf{0}$, which implies that user 1 does not subscribe to the confidential service. Thus, we obtain $\operatorname{rank}\left(\mathbf{W}_{p}\right)=1$. Additionally, following the same procedures in the proof of the second part in Theorem 2, we can show that the energy beamforming matrix yields a rankone solution.

\section{REFERENCES}

[1] Z. Chu, H. X. Nguyen, and G. Caire, "Game theory-based resource allocation for secure WPCN multiantenna multicasting systems," IEEE Trans. Inf. Forensics Security, vol. 13, no. 4, pp. 926-939, Apr. 2018.

[2] R. F. Schaefer and H. Boche, "Physical layer service integration in wireless networks : Signal processing challenges," IEEE Signal Process. Mag., vol. 31, no. 3, pp. 147-156, May 2014.

[3] W. Mei, Z. Chen, L. Li, J. Fang, and S. Li, "On artificial-noise-aided transmit design for multiuser MISO systems with integrated services," IEEE Trans. Veh. Technol., vol. 66, no. 9, pp. 8179-8195, Sept. 2017.

[4] N. Jindal and Z. Q. Luo, "Capacity limits of multiple antenna multicast," in IEEE Int. Symp. Inf. Theory, Seattle, WA, USA, Jul. 2006, pp. 18411845.

[5] N. D. Sidiropoulos, T. N. Davidson, and Z.-Q. Luo, "Transmit beamforming for physical-layer multicasting," IEEE Trans. Signal Process., vol. 54, no. 6, pp. 2239-2251, Jun. 2006.

[6] H. Zhu, N. Prasad, and S. Rangarajan, "Precoder design for physical layer multicasting," IEEE Trans. Signal Process., vol. 60, no. 11, pp. 5932-5947, Nov. 2012.

[7] Y. Liang, H. V. Poor, and S. Shamai, "Information theoretic security," Found. Trends Commun. Inf. Theory, vol. 5, no. 4-5, pp. 355-580, 2009.

[8] A. Khisti and G. W. Wornell, "Secure transmission with multiple antennas I: The MISOME wiretap channel," IEEE Trans. Inf. Theory, vol. 56, no. 7, pp. 3088-3104, Jul. 2010.

[9] A. Khisti and G. W. Wornell, "Secure transmission with multiple antennas II: The MIMOME wiretap channel," IEEE Trans. Inf. Theory, vol. 56, no. 11 , pp. 5515-5532, Nov. 2010. 
[10] Q. Li and W.-K. Ma, "Optimal and robust transmit designs for MISO channel secrecy by semidefinite programming," IEEE Trans. Signal Process., vol. 59, no. 8, pp. 3799-3812, Aug. 2011.

[11] Q. Li and W.-K. Ma, "Spatially selective artificial-noise aided transmit optimization for MISO multi-eves secrecy rate maximization," IEEE Trans. Signal Process., vol. 61, no. 10, pp. 2704-2717, May 2013.

[12] D. W. K. Ng, E. S. Lo, and R. Schober, "Robust beamforming for secure communication in systems with wireless information and power transfer," IEEE Trans. Wireless Commun., vol. 13, no. 8, pp. 4599-4615, Aug. 2014.

[13] Z. Chu, K. Cumanan, Z. Ding, M. Johnston, and S. Le Goff, "Secrecy rate optimizations for a MIMO secrecy channel with a cooperative jammer," IEEE Trans. Veh. Technol., vol. 64, no. 5, pp. 1833-1847, May 2015.

[14] R. Zhang and C. K. Ho, "MIMO broadcasting for simultaneous wireless information and power transfer," IEEE Trans. Wireless Commun., vol. 12, no. 5, pp. 1989-2001, May 2013.

[15] S. Bi, C. K. Ho, and R. Zhang, "Wireless powered communication: opportunities and challenges," IEEE Commun. Mag., vol. 53, no. 4, pp. 117-125, Apr. 2015.

[16] S. Bi, Y. Zeng, and R. Zhang, "Wireless powered communication networks: an overview," IEEE Wireless Commun., vol. 23, no. 2, pp. 10 18, Apr. 2016.

[17] H. Ju and R. Zhang, "Throughput maximization in wireless powered communication networks," IEEE Trans. Wireless Commun., vol. 13, no. 1, pp. 418-428, Jan. 2014.

[18] K. Huang and V. K. N. Lau, "Enabling wireless power transfer in cellular networks: Architecture, modeling and deployment," IEEE Trans. Wireless Commun., vol. 13, no. 2, pp. 902-912, Feb. 2014.

[19] K. Huang and X. Zhou, "Cutting the last wires for mobile communications by microwave power transfer," IEEE Commun. Mag., vol. 53, no. 6, pp. 86-93, Jun. 2015.

[20] C. Zhong, G. Zheng, Z. Zhang, and G. K. Karagiannidis, "Optimum wirelessly powered relaying," IEEE Signal Process. Lett., vol. 22, no. 10, pp. 1728-1732, Oct. 2015.

[21] G. Auer, V. Giannini, C. Desset, I. Godor, P. Skillermark, M. Olsson, M. A. Imran, D. Sabella, M. J. Gonzalez, O. Blume, and A. Fehske, "How much energy is needed to run a wireless network?," IEEE Wireless Commun., vol. 18, no. 5, pp. 40-49, Oct. 2011.

[22] M. Duarte and A. Sabharwal, "Full-duplex wireless communications using off-the-shelf radios: Feasibility and first results," in IEEE ASIMOLAR, Pacific Grove, CA, USA, pp. 1558-1562, Nov. 2010.

[23] D. Nguyen, L. N. Tran, P. Pirinen, and M. Latva-aho, "Precoding for full duplex multiuser MIMO systems: Spectral and energy efficiency maximization," IEEE Trans. Signal Process., vol. 61, no. 16, pp. 40384050, Aug. 2013.

[24] M. Maso, C. F. Liu, C. H. Lee, T. Q. S. Quek, and L. S. Cardoso, "Energy-recycling full-duplex radios for next-generation networks," IEEE J. Sel. Area Commun., vol. 33, no. 12, pp. 2948-2962, Dec. 2015.

[25] Y. Sun, D. W. K. Ng, Z. Ding, and R. Schober, "Optimal joint power and subcarrier allocation for full-duplex multicarrier non-orthogonal multiple access systems," IEEE Trans. Commun., vol. 65, no. 3, pp. 1077-1091, Mar. 2017.

[26] Y. Sun, D. W. K. Ng, J. Zhu, and R. Schober, "Robust and secure resource allocation for full-duplex miso multicarrier noma systems," to appear in IEEE Trans. Commun., 2018.

[27] Y. Zeng and R. Zhang, "Full-duplex wireless-powered relay with selfenergy recycling," IEEE Wireless Commun. Lett., vol. 4, no. 2, pp. 201204, Apr. 2015.

[28] J. Qiao, H. Zhang, X. Zhou, and D. Yuan, "Joint beamforming and time switching design for secrecy rate maximization in wireless-powered FD relay systems," IEEE Trans. Veh. Technol., vol. 67, no. 1, pp. 567-579, Jan. 2018.

[29] G. Chen, P. Xiao, J. R. Kelly, B. Li, and R. Tafazolli, "Full-duplex wireless-powered relay in two way cooperative networks," IEEE Access, vol. 5, pp. 1548-1558, Jan. 2017.

[30] W. Wu, B. Wang, Z. Deng, and H. Zhang, "Secure beamforming for full-duplex wireless powered communication systems with self-energy recycling," IEEE Wireless Commun. Lett., vol. 6, no. 2, pp. 146-149, Apr. 2017.

[31] W. Wu, B. Wang, Y. Zeng, H. Zhang, Z. Yang, and Z. Deng, "Robust secure beamforming for wireless powered full-duplex systems with selfenergy recycling," IEEE Trans. Veh. Technol., vol. 66, no. 11, pp. 1005510069, Nov. 2017.

[32] R. Aslani and M. Rasti, "Distributed power control schemes for in-band full-duplex energy harvesting wireless networks," IEEE Trans. Wireless Commun., vol. 16, no. 8, pp. 5233-5243, Aug. 2017.
[33] I. Csiszár and J. Körner, "Broadcast channels with confidential messages," IEEE Trans. Inf. Theory, vol. 24, pp. 339-348, May 1978.

[34] H. D. Ly, T. Liu, and Y. Liang, "Multiple-input multiple-output gaussian broadcast channels with common and confidential messages," IEEE Trans. Inf. Theory, vol. 56, pp. 5477-5487, Nov Nov. 2010.

[35] R. Liu, T. Liu, H. V. Poor, and S. Shamai, "New results on multipleinput multiple-output broadcast channels with confidential messages," IEEE Trans. Inf. Theory, vol. 59, pp. 1346-1359, March 2013.

[36] E. Ekrem and S. Ulukus, "Capacity region of gaussian MIMO broadcast channels with common and confidential messages," IEEE Trans. Inf. Theory, vol. 58, no. 9, pp. 5669-5680, Sept. 2012.

[37] R. F. Wyrembelski and H. Boche, "Physical layer integration of private, common, and confidential messages in bidirectional relay networks," IEEE Trans. Wireless Commun., vol. 11, no. 9, pp. 3170-3179, Sept. 2012.

[38] R. F. Schaefer and H. Boche, "Robust broadcasting of common and confidential messages over compound channels: Strong secrecy and decoding performance," IEEE Trans. Inf. Forensics Security, vol. 9, no. 10, pp. 1720-1732, Oct. 2014.

[39] A. E. Shafie and N. Al-Dhahir, "Secure communications in the presence of a buffer-aided wireless-powered relay with self-energy recycling," IEEE Wireless Commun. Lett., vol. 5, no. 1, pp. 32-35, Feb. 2016.

[40] A. Mukherjee and A. L. Swindlehurst, "Detecting passive eavesdroppers in the MIMO wiretap channel," in IEEE ICASSP, Tokyo, Japan, Mar. 2012.

[41] S. Boyd and L. Vandenberghe, Convex Optimization. Cambridge, UK: Cambridge University Press, 2004.

[42] D. W. K. Ng, E. S. Lo, and R. Schober, "Multi-objective resource allocation for secure communication in cognitive radio networks with wireless information and power transfer," IEEE Trans. Veh. Technol., vol. 65, no. 5, pp. 3166-3184, May 2016.

[43] Y. Sun, D. W. K. Ng, J. Zhu, and R. Schober, "Multi-objective optimization for robust power efficient and secure full-duplex wireless communication systems," IEEE Trans. Wireless Commun., vol. 15, no. 8 , pp. 5511-5526, Aug. 2016.

[44] A. Charnes and W. W. Cooper, "Programming with linear fractional functionals," Naval Res. Logist. Quarter., vol. 9, pp. 181-186, 1962.

[45] M. Grant and S. Boyd, "CVX: Matlab software for disciplined convex programming." Optimization Methods and Software, Apr. 2012. http: //stanford.edu/ boyd/cvx.

[46] Z.-Q. Luo, J. F. Sturm, and S. Zhang, "Multivariate nonnegative quadratic mappings," SIAM J. Optim., vol. 14, no. 4, pp. 1140-1162, 2004.

[47] M. R. A. Khandaker and K. K. Wong, "Robust secrecy beamforming with energy-harvesting eavesdroppers," IEEE Wireless Commun. Lett., vol. 4, no. 1, pp. 10-13, Feb. 2015.

[48] H. Xing, K. K. Wong, Z. Chu, and A. Nallanathan, "To harvest and jam: A paradigm of self-sustaining friendly jammers for secure AF relaying," IEEE Trans. Signal Process., vol. 63, no. 24, pp. 6616-6631, Dec. 2015.

[49] M. R. A. Khandaker and K. K. Wong, "Masked beamforming in the presence of energy-harvesting eavesdroppers," IEEE Trans. Inf. Forensics Security, vol. 10, no. 1, pp. 40-54, Jan. 2015.

[50] E. Karipidis, N. D. Sidiropoulos, and Z. Q. Luo, "Far-field multicast beamforming for uniform linear antenna arrays," IEEE Trans. Signal Process., vol. 55, no. 10, pp. 4916-4927, Oct. 2007. 